\title{
Gastrointestinal Endoscopy
}

\section{Laparoscopic versus EUS-guided Gastroenterostomy for Gastric Outlet Obstruction: An International Multicentre Propensity Score-Matched Comparison. \\ --Manuscript Draft--}

\begin{tabular}{|c|c|}
\hline Manuscript Number: & GIE-D-20-02527R2 \\
\hline Article Type: & Original Article \\
\hline Keywords: & $\begin{array}{l}\text { Gastric outlet syndrome; laparoscopic gastrojejunostomy; endoscopic ultrasound; } \\
\text { LAMS }\end{array}$ \\
\hline Corresponding Author: & $\begin{array}{l}\text { Schalk van der Merwe, MD, PhD } \\
\text { University Hospitals Leuven: Katholieke Universiteit Leuven Universitaire Ziekenhuizen } \\
\text { Leuven } \\
\text { Leuven, BELGIUM }\end{array}$ \\
\hline First Author: & Michiel Bronswijk, MD \\
\hline \multirow[t]{14}{*}{ Order of Authors: } & Michiel Bronswijk, MD \\
\hline & Giuseppe Vanella \\
\hline & Hannah van Malenstein \\
\hline & Wim Laleman \\
\hline & Joris Jaekers \\
\hline & Baki Topal \\
\hline & Freek Daams \\
\hline & Marc G. Besselink \\
\hline & Paolo Giorgio Arcidiacono \\
\hline & Rogier P. Voermans \\
\hline & Paul Fockens \\
\hline & Alberto Larghi \\
\hline & Roy LJ van Wanrooij \\
\hline & Schalk van der Merwe, MD, $\mathrm{PhD}$ \\
\hline Manuscript Region of Origin: & BELGIUM \\
\hline Abstract: & $\begin{array}{l}\text { Background and aims } \\
\text { In the management of gastric outlet obstruction (GOO), EUS-guided } \\
\text { gastroenterostomy (EUS-GE) seems safe and more effective than enteral stenting. } \\
\text { However, comparisons to laparoscopic gastroenterostomy (L-GE) are scarce. Our aim } \\
\text { was to perform a propensity score-matched comparison between EUS-GE and L-GE. } \\
\text { Methods } \\
\text { An international, multicentre retrospective analysis was performed of consecutive EUS- } \\
\text { GE and L-GE procedures in } 3 \text { academic centers (Jan-2015 to May-2020), using } \\
\text { propensity score-matching in order to minimize selection bias. A standard maximum } \\
\text { propensity score difference of } 0.1 \text { was applied, also considering underlying disease } \\
\text { and oncological staging. } \\
\text { Results } \\
\text { Overall, } 77 \text { patients were treated with EUS-GE and } 48 \text { patients with L-GE. By means of } \\
\text { propensity score-matching, } 37 \text { patients were allocated to both groups, resulting in } 74 \\
\text { (1:1) matched patients. }\end{array}$ \\
\hline
\end{tabular}


Technical success was achieved in 35/37 EUS-GE-treated patients (94.6\%) vs. 100\% in the L-GE group $(p=0.493)$. Clinical success, defined as eating without vomiting or GOO Scoring System $\geq 2$, was achieved in $97.1 \%$ and $89.2 \%$ respectively $(p=0.358)$. Median time to oral intake (1 (IQR 0.3-1.0) vs. 3 (IQR 1.0-5.0) days, $p<0.001)$ and median hospital stay (4 (IQR 2-8) vs 8 (IQR 5.5-20) days, $\mathrm{p}<0.001)$ were significantly shorter in the EUS-GE group. Overall adverse events (AEs) $(2.7 \%$ vs. $27.0 \%, p=0.007)$ and severe AEs $(0.0 \%$ vs. $16.2 \%, p=0.025)$ were identified more frequently in the $L-G E$ group.

\section{Conclusion}

For patients with GOO, EUS-GE and L-GE showed almost identical technical and clinical success. However, reduced time to oral intake, shorter median hospital stay and lower rate of adverse events suggest that the EUS-guided approach might be preferable. 
Contact

Prof.dr.

Schalk Van der Merwe

Schalk.vandermerwe@uzleuven.be tel. +32 16344225

Campus Gasthuisberg

Herestraat 49

3000 Leuven

Belgium

tel. +3216344225
Editor-in-chief

Leuven, February $15^{\text {th }} 2020$
Gastrointestinal endoscopy

Dear prof. Wallace

\section{RE: Response to reviewers}

We hereby thank the editors for considering our revised manuscript entitled, "Laparoscopic versus EUS-guided Gastroenterostomy for Gastric Outlet Obstruction: An International Multicentre Propensity Score-Matched Comparison" for publication in Gastrointestinal Endoscopy.

Secondly, we would like to again express our gratitude towards the reviewers for providing their constructive criticism. Please find a point-by-point answer to Reviewer 1 underneath.

Based on these comments we have adapted the manuscript: changes have been highlighted in light blue for the convenience of the reader.

\section{Response to Reviewer\#1:}

At this time, please check your submitted Disclosure and Attestation form carefully to ensure that it is complete and accurate for ALL authors. GIE takes this very seriously; please be sure all authors have disclosed all conflicts of interest.

Reviewer \#1: The study by Bronswijk encourages the endosonographer to perform gastroenterostomy under EUS.

However, there are a few description on the surgical procedures.

\section{Major}

1. please mention the size of surgical gastrojejunostomy. li is important factor for endosonographers or device company to improve the LAMS in the future.

Response: All laparoscopic gastrojejunostomies were performed using $60 \mathrm{~mm}$ blue Echelon staplers (Johnson \& Johnson, New Brunswick, New Jersey, USA) or 60mm Endo GIA universal staplers (Medtronic Ltd., Dublin, Ireland), with additional staplers and sutures covering the staple line as needed. During construction of the gastro-enteric anastomosis, a 36 French nasogastric tube was temporarily placed through the defect in the majority of cases, in an effort to maintain and confirm patency while stapling or suturing. These considerations were revised in the Methods-section. These procedures 
were performed by expert academic gastrointestinal surgeons specifically credentialed to perform these procedures.

The development of a LAMS is governed by the ability to pass the device along the working channel of the endoscope as well as the ability to deploy the device endoscopically. There will be a trade-off between size of the stent on the one hand, and the functionality of the device on the other hand. As our data have indicated, we have shown that size does matter and that the $20 \mathrm{~mm}$ Axios stent performed better than the $15 \mathrm{~mm}$ stent. However it is conceivable, given the constraints of design, that larger diameter stents will be cumbersome to place and not necessarily aid in better gastric emptying.

\section{Surgical GE showed higher AE rate comparing to EUS-GE. The ratio of surgical GE looks higher than general outcome. Why? How about experience of surgeons? $\mathrm{Pl}$ describe that in the result and discussion.}

Response: Thank you for the in-depth appraisal of our comparative analysis. Indeed, a higher rate of adverse events was seen in the L-GE group, compared to EUS-GE in the overall cohort ( $6.5 \%$ vs. $31.3 \%, p<0.001)$, as well as in the propensity score-matched cohort $(2.7 \%$ vs. $27 \%, p=0.007)$. However, when comparing surgical adverse event rates to the other available studies in this field as well as to the general published literature, we see that our overall surgical adverse event rate is even lower than the study by Perez-Miranda, et al. (41\% surgical adverse events vs. $31.3 \%$ in our study- doi: 10.1097/MCG.0000000000000887) and similar results when compared with the comparative trial by Mouen Kashab, et al. (25\% vs. $31.3 \%$ in our study - doi: $10.1055 / \mathrm{s}$ 0043-101695). This suggests that the superior safety outcomes of EUS-GE observed in our analysis were not due to inferior performance of the surgical comparator group. These comparisons were added to the Discussion-section.

Respectfully,

Schalk Van der Merwe, MD PhD

Prof. Schalk W. van der Merwe MD, MSc, PhD

Associate professor of Medicine

Department of Internal Medicine

Department of Gastroenterology and Hepatology

Division of Liver transplantation

Division of Hepato-pancreaticobiliary interventional endoscopy

Head of the Laboratory of Hepatology, Department of Chronic Diseases, Metabolism and Aging (CROMETA)

University Hospitals Campus Gasthuisberg.

University of Leuven, 
Belgium

schalk.vandermerwe@uzleuven.be

www.lever.be

patricia.caeckaert@uzleuven.be

tel. + 3216344299 - + 3216344626

fax. + 3216344387

UZ Leuven| campus Gasthuisberg | Herestraat 49 | B - 3000 Leuven |www.uzleuven.be 
Dear professor Michael B. Wallace

Editor in Chief - Gastrointestinal Endoscopy

Dear (co-)editors,

We hereby would like to submit our original manuscript entitled 'Laparoscopic versus EUS-guided Gastroenterostomy for Gastric Outlet Obstruction: An International Multicentre Propensity Score-Matched

Campus Gasthuisberg Herestraat 49 3000 Leuven

Belgium

tel. +3216344225
Comparison', by Michiel Bronswijk et al. for publication in Gastrointestinal Endoscopy as an Original Article.

In the management of gastric outlet obstruction (GOO), EUS-guided gastroenterostomy (EUS-GE) has been gaining ground. However, there is still uncertainty regarding the place of EUS-GE in daily clinical practice, due to a lack of data comparing this technique to laparoscopic gastroenterostomy (L-GE) in patients stratified according to potential confounders, such as oncological staging and pre-procedural fragility. We therefore performed a propensity score-matched analysis, comparing EUS-GE with LGE, using a large multicentre international cohort.

There are two reasons why we think that our paper is a great fit for Gastrointestinal Endoscopy. First, our study is one of the largest original cohorts of EUS-GE. And second, using this large international multicentre cohort, the current study represents the first propensity score-matched analysis comparing EUS-GE with LGE. While awaiting high-quality prospective confirmation, these findings may guide gastroenterologists, oncologists and surgeons in therapeutic decision making in the context of GOO.

We thank you for taking our work into consideration and hope that our study merits publication in GIE.

Yours sincerely,

Schalk van der Merwe, MD MSc PhD 


\section{Laparoscopic versus EUS-guided Gastroenterostomy for Gastric Outlet Obstruction: An International Multicentre Propensity Score-Matched Comparison.}

Michiel Bronswijk ${ }^{1,2}$, Giuseppe Vanella ${ }^{3}$, Hannah van Malenstein ${ }^{1}$, Wim Laleman ${ }^{1}$, Joris Jaekers ${ }^{4}$, Baki Topal $^{4}$, Freek Daams ${ }^{5}$, Marc G. Besselink ${ }^{6}$, Paolo Giorgio Arcidiacono ${ }^{3}$, Rogier P. Voermans ${ }^{7}$, Paul Fockens $^{7,8}$, Alberto Larghi ${ }^{9}$, Roy LJ van Wanrooij ${ }^{8}$ and Schalk van der Merwe ${ }^{1}$

\section{Author affiliation:}

1. Department of Gastroenterology and Hepatology, University Hospitals Gasthuisberg, University of Leuven, Leuven, Belgium.

2. Department of Gastroenterology and Hepatology, Imelda General Hospital, Bonheiden, Belgium.

3. Pancreatobiliary Endoscopy and Endosonography Division, IRCCS San Raffaele Scientific Institute, Milan, Italy

4. Department of Visceral Surgery, University Hospitals Gasthuisberg, KU Leuven, Belgium.

5. Department of Surgery, Amsterdam UMC, Vrije Universiteit, Cancer Center Amsterdam, The Netherlands.

6. Department of Surgery, Amsterdam UMC, University of Amsterdam, Cancer Center Amsterdam, The Netherlands.

7. Department of Gastroenterology and Hepatology, Amsterdam UMC, University of Amsterdam, AGEM institute, Amsterdam, The Netherlands

8. Department of Gastroenterology and Hepatology, Amsterdam UMC, Vrije Universiteit Amsterdam, AGEM institute, Amsterdam, The Netherlands

9. Digestive Endoscopy Unit, Fondazione Policlinico Universitario A. Gemelli IRCCS, Università Cattolica del Sacro Cuore, Rome; CERTT, Center for Endoscopic Research Therapeutics and Training, Catholic University, Rome, Italy.

\section{Author statement}

MB was responsible for study design, data collection, statistical analysis and drafting of the manuscript. GV and RvW acted as local lead investigators, collected data, aided in data interpretation and critically revised the final versions of the manuscript. JJ, BT, FD and MGB were responsible for the surgical procedures and revised the final manuscript. GV, HVM, WL, RV, PF and RvW performed the EUS procedures and revised the final version of the manuscript. AL aided in data interpretation and revised the final versions of the manuscript. SVDM was responsible for data interpretation, provided critical insights into study design, performed EUS procedures and critically revised the final version of the manuscript. 


\section{Conflict of interest:}

Michiel Bronswijk received grants from Taewoong, Takeda and Prion Medical. Hannah van Malenstein holds a consultancy agreement with Boston-Scientific. Schalk Van der Merwe holds the Cook chair in Interventional endoscopy and holds consultancy agreements with Cook, Pentax and Olympus. Wim Laleman co-chairs the Boston-Scientific Chair in Therapeutic Biliopancreatic Endoscopy with Schalk Van der Merwe and has consultancy agreements with Boston Scientific and Cook.

Marc G. Besselink received grants for investigator initiated studies from Ethicon, Medtronic and Intuitive. Rogier P. Voermans received a research grant and consultancy fees from Boston Scientific.

The remaining authors declared no potential conflicts of interests regarding the current paper.

\section{Corresponding author:}

Schalk van der Merwe, MD, MSc, PhD

Department of Hepatology

University Hospitals Gasthuisberg, University of Leuven

Herestraat 49 | B- 3000 Leuven |www.uzleuven.be 


\section{Laparoscopic versus EUS-guided Gastroenterostomy for Gastric Outlet Obstruction: An International Multicentre Propensity Score-Matched Comparison.}

Michiel Bronswijk ${ }^{1,2}$, Giuseppe Vanella ${ }^{3}$, Hannah van Malenstein ${ }^{1}$, Wim Laleman ${ }^{1}$, Joris Jaekers ${ }^{4}$, Baki Topal ${ }^{4}$, Freek Daams ${ }^{5}$, Marc G. Besselink ${ }^{6}$, Paolo Giorgio Arcidiacono ${ }^{3}$, Rogier P. Voermans ${ }^{7}$, Paul Fockens $^{7,8}$, Alberto Larghi ${ }^{9}$, Roy LJ van Wanrooij ${ }^{8}$ and Schalk van der Merwe ${ }^{1}$

\section{Author affiliation:}

1. Department of Gastroenterology and Hepatology, University Hospitals Gasthuisberg, University of Leuven, Leuven, Belgium.

2. Department of Gastroenterology and Hepatology, Imelda General Hospital, Bonheiden, Belgium.

3. Pancreatobiliary Endoscopy and Endosonography Division, IRCCS San Raffaele Scientific Institute, Milan, Italy

4. Department of Visceral Surgery, University Hospitals Gasthuisberg, KU Leuven, Belgium.

5. Department of Surgery, Amsterdam UMC, Vrije Universiteit, Cancer Center Amsterdam, The Netherlands.

6. Department of Surgery, Amsterdam UMC, University of Amsterdam, Cancer Center Amsterdam, The Netherlands.

7. Department of Gastroenterology and Hepatology, Amsterdam UMC, University of Amsterdam, AGEM institute, Amsterdam, The Netherlands

8. Department of Gastroenterology and Hepatology, Amsterdam UMC, Vrije Universiteit Amsterdam, AGEM institute, Amsterdam, The Netherlands

9. Digestive Endoscopy Unit, Fondazione Policlinico Universitario A. Gemelli IRCCS, Università Cattolica del Sacro Cuore, Rome; CERTT, Center for Endoscopic Research Therapeutics and Training, Catholic University, Rome, Italy. 


\section{Abstract}

Background and aims:

In the management of gastric outlet obstruction (GOO), EUS-guided gastroenterostomy (EUS-GE) seems safe and more effective than enteral stenting. However, comparisons to laparoscopic gastroenterostomy (L-GE) are scarce. Our aim was to perform a propensity score-matched comparison between EUS-GE and L-GE.

\section{Methods:}

An international, multicentre retrospective analysis was performed of consecutive EUS-GE and L-GE procedures in 3 academic centers (Jan-2015 to May-2020), using propensity score-matching in order to minimize selection bias. A standard maximum propensity score difference of 0.1 was applied, also considering underlying disease and oncological staging.

Results:

Overall, 77 patients were treated with EUS-GE and 48 patients with L-GE. By means of propensity score-matching, 37 patients were allocated to both groups, resulting in 74 (1:1) matched patients.

Technical success was achieved in 35/37 EUS-GE-treated patients (94.6\%) vs. 100\% in the L-GE group ( $p=0.493$ ). Clinical success, defined as eating without vomiting or GOO Scoring System $\geq 2$, was achieved in $97.1 \%$ and $89.2 \%$ respectively ( $p=0.358)$. Median time to oral intake (1 (IQR 0.3-1.0) vs. 3 (IQR 1.0-5.0) days, $p<0.001$ ) and median hospital stay (4 (IQR 2-8) vs 8 (IQR 5.5-20) days, p<0.001) were significantly shorter in the EUS-GE group. Overall adverse events (AEs) $(2.7 \%$ vs. $27.0 \%$, $p=0.007)$ and severe AEs (0.0\% vs. $16.2 \%, p=0.025)$ were identified more frequently in the L-GE group.

Conclusion:

For patients with GOO, EUS-GE and L-GE showed almost identical technical and clinical success. However, reduced time to oral intake, shorter median hospital stay and lower rate of adverse events suggest that the EUS-guided approach might be preferable.

\section{Keywords}

Gastric outlet syndrome, laparoscopic gastrojejunostomy, endoscopic ultrasound, LAMS. 


\section{Introduction}

Gastric outlet obstruction (GOO), defined by a mechanical obstruction of the duodenum, pylorus or antrum, may result from various diseases. The underlying aetiology has shifted from mainly peptic ulcer disease in the past, to predominantly malignant causes at present ${ }^{1,2}$. In the most recent cohorts, GOO was caused by underlying malignancy in up to $85 \%$ of patients, the majority of which could be attributed to pancreatic cancer $3,4,5,6,7$. On the other hand, peptic ulcer disease and chronic pancreatitis are still the most prevalent causes of benign $\mathrm{GOO}^{8,9}$. Consequences of GOO, such as nausea, vomiting, anorexia, need for nasogastric tube decompression and subsequent loss of body mass, may further aggravate this complex clinical entity, increasing morbidity, reducing quality of life and significantly influencing tolerability and efficacy of oncological treatments ${ }^{10}$. Before the advent of endoscopic ultrasound-guided gastroenterostomy (EUS-GE), management of GOO mainly relied on surgical gastroenterostomy, the standard of care for many years ${ }^{11,12}$. Since the late nineties, placement of metal enteral stents was found to provide higher efficacy regarding early re-initiation of oral intake, as well as reduce hospital stay and major adverse events $(A E)^{11}$, albeit at the expense of more recurrent obstructive symptoms due to stent dysfunction ${ }^{13,14}$. Since 2012, EUS-GE has found its way from initial animal studies, into daily practice of tertiary centres ${ }^{15,16}$. This EUS-guided approach has become a minimally invasive alternative for patients with both benign or malignant $\mathrm{GOO}^{3,4}$ and demonstrated higher clinical success and lower need for re-interventions compared to enteral stenting ${ }^{5,17}$. There is still uncertainty regarding the place of EUS-GE in daily clinical practice, due to a lack of data comparing this technique to the current reference standard, laparoscopic gastroenterostomy (L-GE), in patients stratified according to potential confounders, such as oncological staging and pre-procedural fragility ${ }^{18-20}$.

Our aim was to perform a propensity score-matched comparison of EUS-GE to L-GE, using a large retrospective international multicentre cohort. 


\section{Methods}

\section{Patients and study design}

A retrospective analysis was performed of all consecutive L-GE and EUS-GE procedures performed for GOO at the Amsterdam University Medical Centers, location AMC and VUmc (the Netherlands), IRCCS San Raffaele Scientific Institute Milan (Italy) and University Hospitals Leuven (Belgium) between January 2015 and May 2020. For both procedures, identical variables were extracted from patients' electronical medical charts. Inclusion criteria consisted of: (1) symptomatic GOO, (2) endoscopic or radiological confirmation of benign or malignant gastro-duodenal stenosis and (3) treatment with EUS-GE or L-GE. Considering the surgical procedures, only strictly "pure" laparoscopic gastroenterostomies were eligible for inclusion, as to minimize confounding effects of adjunctive procedures, such as metastasectomy, hepatico-enterostomy or cholecystectomy, often performed simultaneously. Each patient gave his or her consent with regards to the procedure. This study was furthermore approved by the central Institutional Review Board (study identifier: s64254) at the University Hospitals Leuven and at each participating center.

\section{Endpoints}

Technical success was defined as the successful creation of a gastro-enteric anastomosis by use of the initially chosen technique (see below for technical considerations). If additional approaches or techniques had to be involved or additional self-expandable metal stents (SEMS) were needed, this was regarded as a technical failure.

Food intake grading was defined using the GOO Scoring System (GOOSS) ( $0=$ no intake, $1=$ liquid only, $2=$ soft solids, $3=$ almost full diet, $4=$ full diet) ${ }^{21,22}$; symptomatic GOO was defined as a GOOSS of 0 . In concordance with previous work ${ }^{7}$, clinical success was defined as eating without vomiting or a GOOSS of $\geq 2$ and was ascertained by evaluation of the electronic patient file, where this parameter is recorded as part of standard practice. Regarding safety, the ASGE lexicon for adverse events was used to stratify AE in mild, moderate, severe or fatal events ${ }^{23}$.

Secondary endpoints were: hospital stay, weight change after 2 months, gastroenterostomy dysfunction, distal obstruction rates, 'time to oral intake' and 'time to full diet' (GOOSS of 4). For EUS-GE procedural time was extracted from the endoscopic electronic reporting system. For L-GE, procedural time was retrieved by revision of the anaesthesiology report, exactly stating the beginning and the end of the procedure. Gastroenterostomy dysfunction was defined as recurrence of obstructive symptoms (GOOSS < 1) after former clinical success, with confirmation of recurrent GOO by endoscopy or imaging studies. Mechanical obstructions located downstream the GE site, without signs of EUS-GE or L-GE dysfunction, were annotated as 'distal obstructions', but not registered as GE dysfunction.

\section{Procedure: the Wireless EUS-gastroenterostomy Simplified Technique (WEST)}

All EUS-GE are performed under deep sedation with propofol or general anaesthesia, using a electrocautery-enhanced lumen apposing metal stent (LAMS) and the Wireless EUS-gastroenterostomy Simplified Technique (WEST) as previously described, under prophylactic antibiotic therapy (Video) ${ }^{24,25}$.

After a 7Fr nasobiliary catheter or enteral feeding tube is placed, through the gastric or duodenal stenosis into the jejunum, water is infused in the targeted loop of small bowel (Figure 1, upper left panel). Using a combination of fluoroscopy and EUSguided identification of the catheter (Figure 1, upper middle panel) the dilated jejunal or enteric loop is accessed using the biflanged electrocautery-enhanced LAMS (Hot-Axios; Boston Scientific, Marlborough, MA, USA). Following successful intraluminal access to the small bowel, the distal flange is deployed under endosonographic guidance; the device is then 
retracted (Figure 1, upper right panel), favouring apposition of the gastric and enteric wall and allowing the opening of the proximal flange inside the endoscope channel (Figure 1, lower left panel); the device is finally pushed outside the working channel together with a careful scope retraction, resulting in deployment of the proximal flange into the gastric lumen. Afterwards, successful deployment is confirmed by either EUS (Figure 1, lower left panel), direct endoscopic visualisation (Figure 2) or, if needed, combined with fluoroscopy and contrast injection (Figure 1, lower middle panel).

\section{Procedure: Laparoscopic gastroenterostomy:}

Following CO2-insufflation (12-15 mmHg intra-abdominal pressure) by a Veress needle or by open introduction, 4-5 trocars are introduced: one camera port around the umbilicus and 3 to 4 trocars at different positions in the upper abdomen. Next, Treitz' ligament is identified and two electrocautery incisions are made, one in the dorsal or anterior gastric wall and one in the jejunum. An anterior, dorsal latero-lateral or side-to-side isoperistaltic gastroenteric anastomosis is constructed. The exact location of the gastroenteric anastomosis, with regards to Treitz' ligament, varies from $30 \mathrm{~cm}$ to $60 \mathrm{~cm}$. During the surgical approach a $60 \mathrm{~mm}$ blue Echelon stapler (Johnson \& Johnson, New Brunswick, New Jersey, USA) or 60mm Endo GIA universal stapler (Medtronic Ltd., Dublin, Ireland) is used depending on the preference of the surgeon, with additional staplers and sutures covering the staple line as needed. During construction of the gastroenteric anastomosis, a 36 French nasogastric tube is temporarily placed through the defect in the majority of cases, in an effort to maintain and confirm patency while stapling or suturing. Anti-traction sutures are used when appropriate. If not in place before the surgical procedure, a nasogastric tube is inserted afterwards and the patient remained on nil per mouth. In this study we only included "pure" L-GE for comparison to EUS-GJ and excluded procedures were adjunctive treatments, such as cholecystectomy, hepaticojejunostomy or metastasectomy were performed. All surgical procedures were performed by gastrointestinal surgeons with extensive experience in laparoscopic gastrointestinal surgery, operating in high-volume academic centres.

\section{Statistical analysis}

Categorical variables were reported as frequencies (\%) and Fisher's test was used to compare these variables. Continuous variables were reported as medians and interquartile range (IQR) or means \pm standard deviation (SD). Student's $t$ test and Mann-Whitney $U$ test were used for comparing continuous variables as appropriate. Differences in outcomes are shown as odds ratio (OR) and 95\% confidence interval (CI). Kaplan-Meier curves were used for overall post-procedural survival analysis, whereas the log-rank test was used for corresponding comparisons. Furthermore, learning curve effect was evaluated by comparing the initial $50 \%$ of procedures with the second half of EUS-GE procedures in each centre. A multiple logistic regression was performed to identify predictors of clinical failure: age, gender, pancreatic cancer, presence of ascites or peritoneal carcinomatosis, use of $15 \mathrm{~mm}$ LAMS and balloon dilation, as well as learning curve were used as variables. P-values $<0.05$ were considered statistically significant.

A propensity score-matched analysis was performed in an effort to minimize selection bias. Age, sex, underlying disease, corresponding disease stage, presence of ascites and presence of peritoneal carcinomatosis were used as variables. The aforementioned variables were selected based on: (1) analysis of previous literature and (2) significant differences between the two groups after univariate analysis. In four previously published studies, which performed a multivariate analysis, peritoneal carcinomatosis and ascites were identified as factors associated with clinical and technical failure respectively3,4,7,26 A standard maximum propensity score difference of 0.1 was admitted for matching.

SPSS version 26.0 (IBM, Chicago, IL, USA) was used for matching and statistical analysis, whereas Graphpad Prism version 9.0.0 for Windows (Graphpad Software, San Diego, CA, USA) was used for the Kaplan-Meier curves and survival analysis. 


\section{Results}

Overall, we identified 126 patients, one of whom was excluded due to missing data, resulting in a total of 77 patients (62\%) who underwent EUS-GE and 48 patients (38\%) undergoing L-GE.

Baseline characteristics are shown in Table 1. Ascites (22.1\% vs. 4.2\%, p=0.009) and pancreatic cancer-induced GOO (48.1\% vs. $29.2 \%, p=0.037$ ) were significantly more frequent in the EUS-GE group, whereas underlying benign disease was identified significantly less (3.9\% vs. 14.6\%, p=0.044) when compared to L-GE-treated patients (Table 1). Technical success (94.8\% (95\% Cl: $87.0-98.4)$ vs. $100 \%$ (95\% Cl: 91.2-100.0), p=0.297) was similar when comparing both groups, while a trend towards higher per-protocol clinical success amongst EUS-GE-treated patients was observed (97.3\% (95\% Cl: 90.0-99.8) vs. 87.5\% (95\% Cl: 74.9-94.5), OR 5.07 (95\% Cl: 0.98-26.28), p=0.057) (Table 2).

Overall, $15 \mathrm{~mm}$ and $20 \mathrm{~mm}$ LAMS were utilized in 11 and 64 patients respectively, with higher clinical success rates $(81.8 \%$ (95\% Cl: $51.2-96.0)$ vs. $100 \%(95 \% \mathrm{Cl}: 93.0-100.0)$ respectively, $\mathrm{p}=0.021)$ and a trend towards shorter median hospital stay (5 days (2.8-11) vs. 4 days (2.0-11), p=0.054) when $20 \mathrm{~mm}$ LAMS were utilized. Intraprocedural balloon dilation of the central part of the LAMS immediately after its placement was performed in $26 \%$ of patients and did not affect efficacy outcomes (Supplementary Table 1). Although two primary clinical failures occurred in patients in whom 15mm LAMS were used without dilation, multivariate analysis did not identify any significant independent risk factors for clinical failure (Supplementary Table 2). Overall AEs (6.5\% (95\% Cl: 2.5-14.7) vs 31.3\% (95\% Cl: 19.9-45.4), OR 0.15 (95\% Cl: 0.05-0.46), p<0.001), and severe AEs (2.6\% (95\% Cl: $0.2-9.5)$ vs $18.8 \%(95 \% \mathrm{Cl}: 10.0-32.2), \mathrm{OR} 0.12(95 \% \mathrm{Cl}: 0.03-0.59), \mathrm{p}=0.007)$ occurred significantly less frequently in EUS-GE versus L-GE treated patients (Table 2).

\section{Propensity score-matched analysis}

By means of propensity score matching, 37 patients were allocated to each group, resulting in a total of 74 (1:1) matched patients (Figure 3). The propensity score-matched cohort revealed an overall mean age of $66.5 \pm 11.8$ years, $44.6 \%$ were female, $36.5 \%$ had underlying pancreatic cancer, with $31.1 \%$ and $6.8 \%$ of patients exhibiting peritoneal carcinomatosis and ascites. Underlying benign disease was present in four (5.4\%) patients. Baseline comparisons between EUS-GE and L-GEtreated patients are shown in Table 3. No significant differences between both study groups were identified.

\section{Efficacy}

In the propensity score-matched EUS-GE group, two technical failures occurred, due to inability to advance the nasobiliary catheter through the stenosis. This resulted in a technical success rate of 94.6\% (35 out of 37 patients, 95\% Cl: 81.4-99.4), compared to $100 \%$ (95\% Cl: 88.8-100.0), p=0.493) amongst L-GE-treated patients (Table 4). Clinical success rates by means of intention to treat analysis (91.9\% (95\% Cl: 78.0-97.9) vs. 89.2\% (95\% Cl: 74.7-96.3), p=1.000), as well as per-protocol analysis were comparable (97.1\% (95\% Cl: 84.2-100.0) vs. 89.2\% (95\% Cl: 74.7-96.3), p=0.358), with primary non-functional surgical gastroenterostomy in three L-GE-patients. Procedure time (46 minutes (IQR 37.5-80.0) vs. 85 minutes (73.0-110), p<0.001), median time to oral intake (1 day (IQR 0.3-1.0) vs. 3 days (IQR 1.0-5.0), p<0.001) and median time to full diet (2 days (IQR 1.03.8) vs. 9 days (IQR 4.0-23), p<0.001) were significantly shorter in the EUS-GE group.

After a median follow-up of 77 days (IQR 27-160) in the EUS group and 123 days in the surgical group (IQR 32-262), gastroenterostomy dysfunction rates (none in both groups) did not differ. With two-months' weight change available in $51.4 \%$ and $56.8 \%$ of patients treated with EUS-GE and L-GE respectively, no significant differences were detected (-0.3kg (IQR -2.41.1) vs. Okg (IQR -3.0-0.7), $p=0.159)$. 


\section{Safety and postoperative outcomes}

The overall number of AEs in the propensity score-matched cohort was lower amongst EUS-GE treated patients (2.7\% (95\% Cl: $0.01-15.1)$ vs. $27 \%$ (95\% Cl: 15.2-43.1), OR 0.07 (95\% Cl: 0.01-0.62), p=0.007) (Table 4). Most AEs in the L-GE group (6 out of 10 ) were severe, mainly consisting of anastomotic leaks $(n=4,10.8 \%)$ or bleeding $(n=2,5.4 \%)$, necessitating surgical reintervention in three patients (8.1\%), while no severe AEs were registered among EUS-GE treated patients $(0.0 \%(95 \% \mathrm{Cl}$ : $0.0-11.2$ ) vs. $16.2 \%$ (95\% Cl: $7.3-31.5)$, OR 0.07 (95\% Cl: $0.00-1.19), p=0.025)$. Mild (2.7\% in each group) and moderate $A E$ rates $(0.0 \%(95 \% \mathrm{Cl}: 0.0-11.2)$ vs. $8.1 \%(95 \% \mathrm{Cl}: 2.1-22.0), \mathrm{p}=0.240)$ were similar in both groups. In two L-GE patients, endoscopic reinterventions was deemed necessary in the context of postoperative bleeding and placement of a transanastomotic stent to treat a dysfunctional surgical anastomosis.

A significantly shorter median hospital stay (4 days (IQR 2.0-8.0) vs. 8 days (IQR 5.5-20), p<0.001) was observed amongst EUSGE-treated patients. Survival analysis did not reveal a significant difference in post-procedural survival in the matched cohort, nor in the overall cohort (Figure 4).

\section{Learning Curve Assessment}

When comparing the first to the second half of both the overall and propensity score-matched EUS-GE cohorts (Supplementary Table 3), no significant differences were found in terms of safety and efficacy. 


\section{Discussion}

In the current analysis, we performed the first propensity score-matched comparison between EUS-GE and L-GE. We found that EUS-GE achieved similar technical and clinical success, with significantly lower overall and severe AEs, faster resumption of oral intake and earlier discharge compared to L-GE. Whilst the EUS-GE technical and clinical success rate was in line with previously published studies $3-9,27$, we observed that technical success depended mainly on the ability to pass a nasobiliary catheter through the stenosis. Furthermore, amongst all technical variables, only LAMS calibre might have some influence on clinical outcome, although this was not confirmed by multivariate analysis.

The paucity of published comparative data makes it difficult to identify a well-defined place for EUS-GE in the management of patients with GOO, especially compared to the more established techniques such as surgery and enteral stenting. Only a single large multicentre retrospective study comparing open gastroenterostomy to EUS-GE has been published so far ${ }^{7}$. The authors reported lower AEs compared to the open surgical approach, at the cost of a lower, but not statistically significant, technical success rate. Surprisingly, length of hospital stay amongst both groups was similar. One can speculate whether this was related to the limited experience available at the time, which may have influenced clinical decisions regarding timing of discharge and general post-EUS-GE management. In our cohort, most patients were discharged after a median of 4 days (IQR 2.0-8.0) compared to 8 days (IQR 5.5-20) in the L-GE group, which may an impact on health care costs and quality of life. We chose to compare our EUS-GE cohort with laparoscopic surgery, as it has proven superior to open surgery, in and outside the context of gastroenterostomy, showing lower morbidity and earlier recovery compared to open surgery, making the L-GE approach the most desired comparator ${ }^{18}$. One previous multicentre retrospective analysis evaluated the efficacy and safety of EUS-GE compared to L-GE, this study only included 25 patients undergoing EUS-GE and did not correct for potential confounding factors or bias ${ }^{19}$. The authors demonstrated increased safety and lower costs using EUS-GE, while retaining similar efficacy to L-GE, even if they detected a non-significant difference in technical success in favour of L-GE ${ }^{19}$

The surgical adverse event rate observed in our study (31.3\%), was similar to those reported by Perez-Miranda, et al (41\%) 19 and Kashab, et al $(25 \%)^{7}$, suggesting that the superior safety outcomes of EUS-GE observed in our analysis were not due to inferior performance of the surgical comparator group. Furthermore, when comparing surgical adverse events rates in historical cohorts of surgical palliative gastroenterostomy, similar $28-30$ or higher ${ }^{31-33}$ adverse event rates were seen when compared to the current analysis.

When considering previous comparisons of EUS-GE with enteral stenting, limited evidence suggests that the latter is associated with a lower rate of clinical success and higher rates of stent failure requiring re-intervention 5,17 . These findings have been confirmed in studies comparing enteral stenting with surgical gastroenterostomy, suggesting that enteral stenting should be considered in the context of very limited life expectancy only13,34.

There are some issues when comparing previous EUS-GE data with our current analysis. First, several techniques have been described for performing EUS-GE, which include the endoscopic ultrasonography-guided double-balloon-occluded gastrojejunostomy bypass (EPASS)-technique, natural orifice transluminal endoscopic surgery (NOTES), rendez-vous methods including balloon-assisted gastroenterostomy, and the direct or 'free-hand'-techniques ${ }^{7,35-37}$. These techniques have been interchangeably used throughout several studies $3,4,7,8,19$, complicating reliable direct comparisons of results. Secondly, several previous papers have published overlapping study cohorts, rendering data interpretation somewhat complicated 4,19,20,35,38. Third, the use of different LAMS with a limited number of studies also including cases where Niti-S Spaxus LAMS (Taewoong Medical Co. Ltd., Ilsan, Korea) were used instead of the Hot-Axios ${ }^{3,7,26}$. Regarding different EUS-GE approaches, a comparative study has shown that the direct method achieves similar technical and clinical success, with a similar safety profile when 
compared to balloon-assisted EUS-GE ${ }^{3}$. However, in the context of the direct method, procedure time was more than twice shorter (35.7 vs. 89.9min, $\mathrm{p}<0.001$ ), thus suggesting the direct technique as the preferred method. In all of our patients, only the Wireless EUS-gastroenterostomy Simplified Technique (WEST) was used, indicating that there is no need for a 19G 'finder'-needle, as has been described in the direct technique, or a guidewire and balloon in order to perform EUS-GE safely and effectively ${ }^{3,24,25}$. We recommend against inflating the targeted loop of small bowel by water or contrast injection using a 19-gauge needle, such as in the setting of EUS-directed transenteric ERCP (EDEE) ${ }^{39}$, an approach which may carry a higher theoretical risk of puncturing a more distal enteric loop or even the colon. We do recommend using the most straightforward technique available, in an effort to reduce the number of additional accessories requiring exchange, which in our opinion carries an increased risk of adverse events by complicating positioning, visualisation and, as time passes, reduction in small bowel distention.

While the usefulness of EUS-GE in malignant disease has been reported in various studies, the evidence of this procedure in benign disease has been increasing since only recently5,7,17. Doubts concerning LAMS patency and long-term results have led to restricted use in benign diseases. In 2020, James et al. published their series on EUS-GE in benign disease, revealing that surgery was averted in $83.3 \%$ of patients and regression of the benign stricture allowed for LAMS removal in the majority of patients over time ${ }^{9}$. Together with long-term follow-up data published in $2019^{6}$, which showed a $15 \%$ recurrence rate after a median follow-up time of 169 (malignant disease) and 319.5 days (benign disease), we can conclude that especially in patients with malignant disease GOO, recurrence is an issue. In our current propensity score-matched analysis gastroenterostomy dysfunction did not occur at all, although two cases of distal enteric obstruction, due to metastatic peritoneal disease, were identified in the EUS-GE group, compared to one in the L-GE-group.

In comparison to various other studies, where there was no mention of the incidence of ascites $3,4,6-9,17,19,21 \%$ of our patients underwent EUS-GE despite the presence of ascites, without any significant related AEs. Although ascites has been regarded as a strict contraindication for EUS-GE, these results, together with a retrospective analysis in $2019^{5}$, suggest that patients with mild or localized ascites can be considered for EUS-GE without risking leakage or subsequent peritonitis, provided that there is no tense ascites and that the LAMS trajectory is not compromised due to fluid interference.

\section{Limitations and strengths:}

Several limitations of the current study should be addressed. First, the retrospective nature of this analysis might have inadvertently introduced some bias. Secondly, due to the study design, a certain degree of missing data was identified, most especially in the context of body weight evolution. Third, generalizability of our data might be an issue, as all endoscopists were highly trained and operating in high-volume settings.

We have tried to address some of these limitations in our study design. We included a propensity score-matched design, correcting for the selected variables to limit selection bias. These specific variables were chosen as they were differently distributed amongst the two treatment groups, whilst potentially influencing technical and clinical success, as well as 'time to oral intake', 'time to full diet', overall survival and gastroenterostomy dysfunction rates. One of the disadvantages of propensity score-matching, is the dependence on the matching criteria. We therefore included various variables as to provide a stringent matching process. To reduce larger treatment effects and higher degrees of bias of single centre studies, we involved three different tertiary referral centres recruiting similar patients and all performing EUS-GE using the WEST approach $^{24,25}$. Finally, with 77 patients in whom EUS-GE was performed, our study is one of the largest published original cohorts of EUS-GE, and the largest study to date to compare EUS-GE with L-GE. 
In conclusion, this study suggests that in patients with gastric outlet syndrome, EUS-GE and L-GE provide almost identical technical and clinical success rates. Lower time to oral intake, shorter hospital stay and a lower rate of adverse events prudentially suggest that EUS-GE should be the preferred approach in patients with GOO. While awaiting high-quality prospective confirmation, these findings should guide gastroenterologists, oncologists and surgeons in considering EUS-GE for treating GOO, especially in the setting of malignancy, where patients will benefit from the least invasive technique with the highest expected efficacy.

\section{Acknowledgements}

None

\section{References}

1. McCarty TR, Garg R, Thompson CC, Rustagi T. Efficacy and safety of EUS-guided gastroenterostomy for benign and malignant gastric outlet obstruction: a systematic review and meta-analysis. Endosc Int Open 2019;7:E1474-E1482.

2. Shone DN, Nikoomanesh P, Smith-Meek MM et al. Malignancy is the most common cause of gastric outlet obstruction in the era of H2 blockers. Am J Gastroenterol 1995;90:1769-1770

3. Chen YI, Kunda R, Storm AC, et al. EUS-guided gastroenterostomy: a multicenter study comparing the direct and balloonassisted techniques. Gastrointest Endosc 2018;87:1215-21.

4. Tyberg A, Perez-Miranda M, Sanchez-Ocaña R, et al. Endoscopic ultrasound-guided gastrojejunostomy with a lumenapposing metal stent: a multicenter, international experience. Endosc Int Open 2016;4:E276-81.

5. Ge PS, Young JY, Dong W, et al. EUS-guided gastroenterostomy versus enteral stent placement for palliation of malignant gastric outlet obstruction. Surg Endosc 2019;33:3404-11.

6. Kerdsirichairat T, Irani S, Yang J, et al. Durability and long-term outcomes of direct EUS-guided gastroenterostomy using lumen-apposing metal stents for gastric outlet obstruction. Endosc Int Open 2019;7:E144-E150.

7. Khashab MA, Bukhari M, Baron TH, et al. International multicenter comparative trial of endoscopic ultrasonographyguided gastroenterostomy versus surgical gastrojejunostomy for the treatment of malignant gastric outlet obstruction. Endosc Int Open 2017;5:E275-E281

8. Chen YI, James TW, Agarwal A, et al. EUS-guided gastroenterostomy in management of benign gastric outlet obstruction. Endosc Int Open 2018;6:E363-E368.

9. James TW, Greenberg S, Grimm IS, Baron TH. EUS-guided gastroenteric anastomosis as a bridge to definitive treatment in benign gastric outlet obstruction. Gastrointest Endosc 2020;91:537-542.

10. Klute KA, Brouwer J, Jhawer M, et al. Chemotherapy dose intensity predicted by baseline nutrition assessment in gastrointestinal malignancies: A multicentre analysis. Eur J Cancer 2016;63:189-200. 
11. Ly J, O'Grady G, Mittal A, et al. A systematic review of methods to palliate malignant gastric outlet obstruction. Surg Endosc 2010;24:290-297.

12. Wilson RG, Varma JS. Laparoscopic gastroenterostomy for malignant duodenal obstruction. Br J Surg 1992;79:1348.

13. Jeurnink SM, Van Eijck CHJ, Steyerberg EW, et al. Stent versus gastrojejunostomy for the palliation of gastric outlet obstruction: a systematic review. BMC Gastroenterol 2007;7:1-10.

14. Khashab M, Alawad AS, Shin EJ, et al. Enteral stenting versus gastrojejunostomy for palliation of malignant gastric outlet obstruction. Surg Endosc 2013;27:2068-2075.

15. Binmoeller KF, Shah JN. Endoscopic ultrasound-guided gastroenterostomy using novel tools designed for transluminal therapy: a porcinestudy. Endoscopy 2012;44:499-503

16. Itoi T, Ishii K, Tanaka R, et al. Current status and perspective of endoscopicultrasonography-guided gastrojejunostomy: endoscopic ultrasonography-guided double-balloon-occluded gastrojejunostomy (with videos). J Hepatobiliary Pancreat Sci 2015;22:3-11

17. Chen YI, Itoi T, Baron TH, et al. EUS-guided Gastroenterostomy is comparable to enteral stenting with fewer reinterventions in malignant gastric outlet obstruction. Surg Endosc 2017;31:2946-2952.

18. Al-Rashedy M, Dadibhai M, Shareif A, et al. Laparoscopic gastric bypass for gastric outlet obstruction is associated with smoother, faster recovery and shorter hospital stay compared with open surgery. J Hepatobiliary Pancreat Surg $2005 ; 12: 474-478$.

19. Perez-Miranda M, Tyberg A, Poletto D, et al. EUS-guided gastrojejunostomy versus laparoscopic gastrojejunostomy: an international collaborative study. J Clin Gastroenterol 2017;51:896-899.

20. Khashab MA, Kumbhari V, Grimm IS, et al. EUS-guided gastroenterostomy: the first U.S. clinical experience (with video). Gastrointest Endosc 2015;82:932-938.

21. Brewer Gutierrez OI, Nieto J, Irani S, et al. Double endoscopic bypass for gastric outlet obstruction and biliary obstruction. Endosc Int Open 2017;5:E893-E899.

22. Adler DG, Baron TH. Endoscopic palliation of malignant gastric outlet obstruction using self-expanding metal stents: experience in 36 patients. Am J Gastroenterol 2002;97:72-78.

23. Cotton PB, Eisen GM, Aabakken L, et al. A lexicon for endoscopic adverse events: report of an ASGE workshop. Gastrointest Endosc 2010;71:446-454.

24. Bronswijk M, Fransen L, Vanella G, et al. Successful treatment of superior mesenteric artery syndrome by endoscopic ultrasound-guided gastrojejunostomy [published online ahead of print, 2020 Jun 19]. Endoscopy 2020;10.1055/a-11903228.

25. Bronswijk M, Vanella G, Petrone MC, et al. EUS-guided gastroenterostomy: Less is more! The wireless EUS-guided gastroenterostomy simplified technique. VideoGIE 2020;5:442. 
26. Wannhoff A, Ruh N, Meier B, et al. Endoscopic gastrointestinal anastomoses with lumen-apposing metal stents: predictors of technical success [published online ahead of print, 2020 May 1]. Surg Endosc 2020;10.1007/s00464-02007594-5.

27. Antonelli G, Kovacevic B, Karstensen JG, et al. Endoscopic ultrasound-guided gastro-enteric anastomosis: A systematic review and meta-analysis. Dig Liver Dis 2020;52:1294-1301.

28. Bednarsch J, Czigany Z, Heise D, et al. Influence of peritoneal carcinomatosis on perioperative outcome in palliative gastric bypass for malignant gastric outlet obstruction - a retrospective cohort study. World J Surg Oncol 2020;18:25.

29. Denley SM, Moug SJ, Carter CR, McKay CJ. The outcome of laparoscopic gastrojejunostomy in malignant gastric outlet obstruction. Int J Gastrointest Cancer 2005;35:165-169.

30. Kazanjian KK, Reber HA, Hines OJ. Laparoscopic gastrojejunostomy for gastric outlet obstruction in pancreatic cancer. Am Surg 2004;70:910-913.

31. Jeurnink SM, Steyerberg EW, Hof Gv, et al. Gastrojejunostomy versus stent placement in patients with malignant gastric outlet obstruction: a comparison in 95 patients. J Surg Oncol 2007;96:389-396.

32. Zhang LP, Tabrizian P, Nguyen S, et al. Laparoscopic gastrojejunostomy for the treatment of gastric outlet obstruction. JSLS 2011;15:169-173.

33. Kuriansky J, Sáenz A, Astudillo E, et al. Simultaneous laparoscopic biliary and retrocolic gastric bypass in patients with unresectable carcinoma of the pancreas. Surg Endosc 2000;14:179-181.

34. Jeurnink SM, Steyerberg EW, van Hooft JE, et al. Surgical gastrojejunostomy or endoscopic stent placement for the palliation of malignant gastric outlet obstruction (SUSTENT study): a multicenter randomized trial. Gastrointest Endosc 2010;71:490-9.

35. Itoi T, Ishii K, Ikeuchi N, et al. Prospective evaluation of endoscopic ultrasonography-guided double-balloon-occluded gastrojejunostomy bypass (EPASS) for malignant gastric outlet obstruction. Gut 2016;65:193-195.

36. Irani S, Baron TH, Itoi T, Khashab MA. Endoscopic gastroenterostomy: techniques and review. Curr Opin Gastroenterol 2017;33(5):320-329.

37. Barthet M, Binmoeller KF, Vanbiervliet G, et al. Natural orifice transluminal endoscopic surgery gastroenterostomy with a biflanged lumenapposing stent: first clinical experience (with videos). Gastrointest Endosc 2015;81:215-218.

38. Khashab MA, Baron TH, Binmoeller KF, et al. EUS-guided gastroenterostomy: a new promising technique in evolution. Gastrointest Endosc 2015;81:1234-1236.

39. Ichkhanian Y, Yang J, James TW, et al. EUS-directed transenteric ERCP in non-Roux-en-Y gastric bypass surgical anatomy patients (with video). Gastrointest Endosc 2020;91:1188-1194. 


\section{Figure legend}

Figure 1. Stepwise approach to the Wireless EUS-gastroenterostomy Simplified Technique (WEST).

Upper left: Fluoroscopic image, placement of a 7Fr nasobiliary catheter or enteral feeding tube through the gastric or duodenal stenosis into the jejunum. Confirmation of the distal position by contrast opacification.

Upper middle: Endoscopic ultrasound image, showing distention of the targeted loop of small bowel after infusion of water. Upper right: Using a combination of fluoroscopy and EUS-guided identification of the catheter, the dilated jejunal or enteric loop is accessed using the biflanged electrocautery-enhanced LAMS, after which the distal flange is deployed under endosonographic guidance. The device is then retracted onto the gastric wall.

Lower left: Endoscopic ultrasound image, after compressing the gastric and enteric wall, as well as deployment of the proximal flange inside the endoscope channel, the device is finally pushed outside the working channel together with a careful scope retraction, resulting in deployment of the proximal flange into the gastric lumen.

Lower middle: Fluoroscopic evaluation after LAMS release, showing the successful deployment of the LAMS between the stomach and small bowel.

Lower right: Endoscopic view, after recannulation with a diagnostic catheter.

Figure 2. Direct endoscopic visualisation of successful gastroenterostomy.

Figure 3. Study flowchart.

Figure 4. Post-procedural survival analysis. 


\section{Tables}

Table 1. Overall baseline characteristics.

\begin{tabular}{|c|c|c|c|c|c|}
\hline & $\begin{array}{c}\text { EUS-GE } \\
(n=77)\end{array}$ & & $\begin{array}{c}\text { L-GE } \\
(n=48)\end{array}$ & & $P$ value \\
\hline \multicolumn{6}{|l|}{ Variable } \\
\hline Age (years), mean \pm SD & 65 & \pm 12.3 & 66 & \pm 11.6 & 0.478 \\
\hline Female, $\mathrm{n}(\%)$ & 36 & $(46.8 \%)$ & 19 & (39.6\%) & 0.432 \\
\hline Median follow up duration, days (IQR) & 76 & $(36-136)$ & 122 & $(35-274)$ & 0.057 \\
\hline \multicolumn{6}{|l|}{ Primary disease } \\
\hline Pancreatic cancer & 37 & $(48.1 \%)$ & 14 & $(29.2 \%)$ & 0.037 \\
\hline Biliary/gallbladder cancer & 9 & $(11.7 \%)$ & 2 & $(4.2 \%)$ & 0.149 \\
\hline Gastric cancer & 7 & $(9.1 \%)$ & 5 & $(10.4 \%)$ & 0.807 \\
\hline Duodenal cancer & 11 & $(14.3 \%)$ & 10 & $(20.8 \%)$ & 0.341 \\
\hline Breast cancer & 2 & $(2.6 \%)$ & 2 & $(4.2 \%)$ & 0.463 \\
\hline Colorectal cancer & 2 & $(2.6 \%)$ & 1 & $(2.1 \%)$ & 1.000 \\
\hline NET & 1 & $(1.3 \%)$ & 0 & $(0.0 \%)$ & 0.384 \\
\hline Ampullary cancer & 0 & $(0.0 \%)$ & 1 & $(2.1 \%)$ & 0.384 \\
\hline NSCLC & 3 & $(3.9 \%)$ & 1 & $(2.1 \%)$ & 1.000 \\
\hline Benign disease & 3 & $(3.9 \%)$ & 7 & $(14.6 \%)$ & 0.044 \\
\hline \multicolumn{6}{|l|}{ Disease stage } \\
\hline Local invasion & 25 & $(32.5 \%)$ & 13 & $(27.1 \%)$ & 0.555 \\
\hline Liver metastases & 8 & $(10.4 \%)$ & 9 & $(18.8 \%)$ & 0.193 \\
\hline Peritoneal metastastases & 8 & $(10.4 \%)$ & 10 & $(20.8 \%)$ & 0.122 \\
\hline Diffuse metastatic & 19 & $(24.7 \%)$ & 9 & $(18.8 \%)$ & 0.512 \\
\hline \multicolumn{6}{|l|}{ Disease manifestations } \\
\hline Ascites & 17 & $(22.1 \%)$ & 2 & $(4.2 \%)$ & 0.009 \\
\hline Peritoneal carcinomatosis & 20 & $(26.0 \%)$ & 16 & (33.3\%) & 0.420 \\
\hline
\end{tabular}


Table 2. Overall outcomes.

1

\begin{tabular}{|c|c|c|c|c|c|}
\hline & $\begin{array}{c}\text { EUS-GE } \\
(n=77)\end{array}$ & & $\begin{array}{c}\text { L-GE } \\
(n=48)\end{array}$ & & OR $(95 \% \mathrm{Cl}), P$ value \\
\hline \multicolumn{6}{|l|}{ Efficacy } \\
\hline Technical success, n (\%) & 73 & $(94.8 \%)$ & 48 & $(100 \%)$ & $0.17(0.01-3.20), 0.297$ \\
\hline Clinical success, n (\%) & 71 & $(92.2 \%)$ & 42 & $(87.5 \%)$ & $1.69(0.51-5.58), 0.534$ \\
\hline Per protocol clinical success, n (\%) & 71 & $(97.3 \%)$ & 42 & $(87.5 \%)$ & $5.07(0.98-26.28), 0.057$ \\
\hline Median time to oral intake, days (IQR) & 1 & $(0-1)$ & 3 & $(1-5)$ & $<0.001$ \\
\hline Full diet tolerability, n (\%) & 32 & $(41.6 \%)$ & 19 & (39.6\%) & $1.16(0.56-2.44), 0.854$ \\
\hline Median time to full diet, days (IQR) & 2 & $(1-4)$ & 8 & $(4-21)$ & $<0.001$ \\
\hline Gastroenterostomy dysfunction, n (\%) & 1 & $(1.3 \%)$ & 0 & $(0.0 \%)$ & $1.90(0.08-47.64), 1.000$ \\
\hline Median time to dysfunction, days (IQR) & 243 & N/A & N/A & N/A & N/A \\
\hline Distal obstruction, n (\%) & 8 & $(10.4 \%)$ & 1 & $(1.3 \%)$ & $5.45(0.66-45.02), 0.151$ \\
\hline Median time to distal obstruction, days (IQR) & 34 & $(18-138)$ & 13 & N/A & $\mathrm{N} / \mathrm{A}$ \\
\hline \multicolumn{6}{|l|}{ Safety } \\
\hline Overall adverse events, n (\%) & 5 & $(6.5 \%)$ & 15 & $(31.3 \%)$ & $0.15(0.05-0.46),<0.001$ \\
\hline Mild, $n(\%)$ & 2 & $(2.6 \%)$ & 1 & $(2.1 \%)$ & $1.25(0.11-14.21), 1.000$ \\
\hline Post-procedural fever, $\mathrm{n}(\%)$ & 2 & $(2.6 \%)$ & 0 & $(0.0 \%)$ & $3.21(0.15-68.35), 0.523$ \\
\hline Moderate, $\mathrm{n}(\%)$ & 1 & $(1.3 \%)$ & 5 & $(10.4 \%)$ & $0.11(0.01-1.00), 0.106$ \\
\hline Sepsis, n (\%) & 1 & $(1.3 \%)$ & 0 & $(0.0 \%)$ & $1.90(0.08-47.64), 1.000$ \\
\hline \multirow[t]{2}{*}{ Need for re-endoscopy, n (\%) } & 0 & $(0.0 \%)$ & 4 & $(8.3 \%)$ & $0.06(0.00-1.21), 0.020$ \\
\hline & 2 & $(2.6 \%)$ & 9 & $(18.8 \%)$ & $0.12(0.03-0.59), 0.007$ \\
\hline Intra-peritoneal LAMS deployment, n (\%) & 2 & $(2.6 \%)$ & N/A & $N / A$ & $N / A$ \\
\hline Anastomotic leak, n (\%) & N/A & N/A & 3 & $(6.3 \%)$ & $N / A$ \\
\hline Anastomotic bleeding, $\mathrm{n}(\%)$ & N/A & N/A & 2 & $(4.2 \%)$ & $\mathrm{N} / \mathrm{A}$ \\
\hline Surgical re-intervention, n (\%) & N/A & N/A & 3 & $(6.3 \%)$ & N/A \\
\hline Fatal, $\mathrm{n}(\%)$ & 0 & $(0.0 \%)$ & 0 & $(0.0 \%)$ & 1.000 \\
\hline \multicolumn{6}{|l|}{ Other } \\
\hline Median procedure duration, min (IQR) & 51 & $(36-79.8)$ & 95 & $(75-118)$ & $<0.001$ \\
\hline Median hospital stay, days (IQR) & 4 & $(2-10.5)$ & 8 & $(5-20)$ & $<0.001$ \\
\hline Median weight change after 2 months, kg (IQR) & -1 & $(-4.0-1.1)$ & $-0,4$ & $(-4.2-0.8)$ & 0.390 \\
\hline Median post-procedural survival, days (IQR) & 103 & $(44-252)$ & 147 & $(68-335)$ & 0.246 \\
\hline
\end{tabular}

Abbreviations: Cl: confidence interval, EUS-GE: Endoscopic ultrasound-guided gastroenterostomy, IQR: interquartile range, LGE: laparoscopic gastroenterostomy, N/A: not applicable, OR: odds ratio. 
Table 3. Matched cohort: baseline characteristics.

\begin{tabular}{|c|c|c|c|c|c|}
\hline & $\begin{array}{c}\text { EUS-GE } \\
(n=37)\end{array}$ & & $\begin{array}{c}\text { L-GE } \\
(n=37)\end{array}$ & & $P$ value \\
\hline \multicolumn{6}{|l|}{ Variable } \\
\hline Age (years), mean \pm SD & 66.5 & \pm 12.5 & 66.4 & \pm 11.1 & 0.954 \\
\hline Female, $\mathrm{n}(\%)$ & 18 & $(48.7 \%)$ & 15 & $(40.5 \%)$ & 0.640 \\
\hline Median follow up duration, days (IQR) & 77 & $27-160$ & 123 & $32-262$ & 0.105 \\
\hline \multicolumn{6}{|l|}{ Primary disease } \\
\hline Pancreatic cancer & 15 & $(40.5 \%)$ & 13 & $(35.1 \%)$ & 0.811 \\
\hline Biliary/gallbladder cancer & 5 & $(13.5 \%)$ & 2 & $(5.4 \%)$ & 0.430 \\
\hline Gastric cancer & 5 & $(13.5 \%)$ & 5 & $(13.5 \%)$ & 1.000 \\
\hline Duodenal cancer & 6 & $(16.2 \%)$ & 8 & $(21.6 \%)$ & 0.768 \\
\hline Benign disease & 2 & $(5.4 \%)$ & 2 & $(5.4 \%)$ & 1.000 \\
\hline Breast cancer & 1 & $(2.7 \%)$ & 1 & $(2.7 \%)$ & 1.000 \\
\hline Colorectal cancer & 2 & $(5.4 \%)$ & 0 & $(0.0 \%)$ & 0.493 \\
\hline NET & 1 & $(2.7 \%)$ & 0 & $(0.0 \%)$ & 1.000 \\
\hline Ampullary cancer & 0 & $(0.0 \%)$ & 1 & $(2.7 \%)$ & 1.000 \\
\hline NSCLC & 1 & $(2.7 \%)$ & 1 & $(2.7 \%)$ & 1.000 \\
\hline \multicolumn{6}{|l|}{ Disease stage } \\
\hline Local invasion & 15 & $(40.5 \%)$ & 12 & $(32.4 \%)$ & 0.630 \\
\hline Liver metastases & 6 & $(16.2 \%)$ & 8 & $(21.6 \%)$ & 0.768 \\
\hline Peritoneal metastastases & 6 & $(16.2 \%)$ & 7 & $(18.9 \%)$ & 1.000 \\
\hline Diffuse metastatic & 8 & $(21.6 \%)$ & 6 & $(16.2 \%)$ & 0.768 \\
\hline \multicolumn{6}{|l|}{ Disease manifestations } \\
\hline Ascites & 3 & (8.1\%) & 2 & $(5.4 \%)$ & 1.000 \\
\hline Peritoneal carcinomatosis & 10 & $(27.0 \%)$ & 13 & $(35.1 \%)$ & 0.616 \\
\hline
\end{tabular}

1

2

3 
Table 4. Matched cohort: outcome comparisons.

1

2

\section{EUS-GE \\ $(n=37)$ \\ L-GE \\ $(n=37)$}

\section{Efficacy}

Technical success, n (\%)

Clinical success, $n(\%)$

\author{
35
}

34

(91.9\%)

33

$(100 \%$

(89.2\%)

Per protocol clinical success, $n(\%)$

$34 / 35$

(97.1\%)

(89.2\%)

Median time to oral intake, days (IQR)

Full diet at discharge, $n(\%)$

(0.3-1.0)

(1.0-5.0)

$(56.8 \%)$

(37.8\%)

Median time to full diet, days (IQR)

Gastroenterostomy dysfunction, $\mathrm{n}(\%)$

Distal obstruction, $\mathrm{n}(\%)$

\section{Safety}

Overall adverse events, $\mathrm{n}(\%)$

Mild, n(\%)

Moderate, $\mathrm{n}(\%)$

Severe, $\mathrm{n}(\%)$

Endoscopic reintervention

(1.0-3.8)

(4.0-23)

$(0.0 \%)$

$(0.0 \%)$

$(5.4 \%)$

(2.7\%)
(94.6\%) - 37

\section{OR (95\% Cl), $P$ value}

0.19 (0.01-4.08), 0.493

1.37 (0.29-6.62), 1.000

$4.12(0.44-38.83), 0.358$

$<0.001$

$2.16(0.85-5.46), 0.162$

$<0.001$

1.000

$2.06(0.18-23.72), 1.000$

0.07 (0.01-0.62), 0.007 1.000

$0.13(0.01-2.64), 0.240$

$0.19(0.01-4.08), 0.493$

$0.07(0.00-1.19), 0.025$

$0.13(0.01-2.64), 0.240$

$0.10(0.01-1.91), 0.115$

$\begin{array}{lllll}\text { Anastomotic leak } & 0 & (0.0 \%) & 4 & (10.8 \%)\end{array}$

(5.4\%)

(0.0\%)
$0.19(0.01-4.08), 0.493$

1.000

(0.0\%) $\quad 0$

Fatal, $\mathrm{n}(\%)$

(37.5-80) $\quad 85$

(73.0-110)

$<0.001$ Median hospital stay, days (IQR) (2.0-8.0) $\quad 8$

$<0.001$ Median 2-months' weight change, kg (IQR) $-0,3$

0.159

Median post-procedural survival, days (IQR) 96

(41.5-248) $\quad 152$

(43.5-282)

0.317 


\section{Supplementary tables}

Supplementary table 1 . Sub-analysis comparing $15 \mathrm{~mm}$ and $20 \mathrm{~mm}$ LAMS efficacy and outcomes with or without balloon dilation.

\begin{tabular}{|c|c|c|c|c|c|}
\hline Variable: & $\begin{array}{c}15 \mathrm{~mm} \text { LAMS } \\
(\mathrm{n}=11)\end{array}$ & & $\begin{array}{c}20 \mathrm{~mm} \text { LAMS } \\
(\mathrm{n}=64)\end{array}$ & & $P$-value \\
\hline Technical success, n (\%) & $11 / 11$ & $100 \%$ & $62 / 64$ & $96.9 \%$ & 1.000 \\
\hline Clinical success*, n (\%) & $9 / 11$ & $81.8 \%$ & $62 / 62$ & $100 \%$ & 0.021 \\
\hline Median time to oral intake, days (IQR) & 1 & $(0-1)$ & 1 & $(0-1)$ & 0.826 \\
\hline Full diet tolerability, $\mathrm{n}(\%)$ & 6 & $54.5 \%$ & 45 & $70.3 \%$ & 0.314 \\
\hline Median time to full diet, days (IQR) & 2 & $(1-3)$ & 2 & $(1-4)$ & 0.099 \\
\hline Overall adverse events, n (\%) & 1 & $9.1 \%$ & 4 & $6.5 \%$ & 0.558 \\
\hline Gastroenterostomy dysfunction, n (\%) & 0 & $0.0 \%$ & 1 & $1.6 \%$ & 1.000 \\
\hline Median time to dysfunction, days (IQR) & N/A & N/A & 243 & N/A & N/A \\
\hline Median hospital stay, days (IQR) & 5 & $(2.8-11)$ & 4 & $(2.0-11)$ & 0.054 \\
\hline Median weight change after 2 months, kg (IQR) & $-1,4$ & $(-5.2-0.6)$ & -1 & $(-4.0-1.1)$ & 0.430 \\
\hline Variable: & $\begin{array}{l}\text { With LAMS } \\
\text { dilation }(n=20)\end{array}$ & & $\begin{array}{l}\text { Without LAMS } \\
\text { dilation }(n=55)\end{array}$ & & $P$-value \\
\hline Technical success, n (\%) & $20 / 20$ & $100 \%$ & $53 / 55$ & $96.4 \%$ & 1.000 \\
\hline Clinical success*, n (\%) & $20 / 20$ & $100 \%$ & $51 / 53$ & $96.2 \%$ & 1.000 \\
\hline Median time to oral intake, days (IQR) & 1 & $(0-1)$ & 1 & $(0-1)$ & 0.153 \\
\hline Full diet tolerability, $\mathrm{n}(\%)$ & 10 & $50.0 \%$ & 39 & $70.9 \%$ & 0.107 \\
\hline Median time to full diet, days (IQR) & 2.5 & $(1-4)$ & 2 & $(2-8)$ & 0.254 \\
\hline Overall adverse events, n (\%) & 1 & $5.0 \%$ & 4 & $7.3 \%$ & 0.579 \\
\hline Gastroenterostomy dysfunction, n (\%) & 1 & $5.0 \%$ & 0 & $0.0 \%$ & 1.000 \\
\hline Median time to dysfunction, days (IQR) & 243 & N/A & $\mathrm{N} / \mathrm{A}$ & $\mathrm{N} / \mathrm{A}$ & N/A \\
\hline Median hospital stay, days (IQR) & 5 & $(2.0-11)$ & 4 & $(2.0-11)$ & 0.022 \\
\hline Median weight change after 2 months, kg (IQR) & -1.4 & $(-5.1-0.7)$ & -1 & $(-4.0-1.1)$ & 0.849 \\
\hline
\end{tabular}

Abbreviations: IQR: interquartile range, LAMS: lumen-apposing metal stent, kg: kilogram, N/A: not applicable.

*: per-protocol analysis. 
Supplementary table 2. Multivariate analysis of variables in EUS-GE clinical failure.

1

2

\begin{tabular}{lccc}
\hline Variable & OR & $95 \% \mathrm{Cl}$ & $P$-value \\
\hline Age & 0.98 & $0.90-1.03$ & 0.532 \\
Gender, female & 2.95 & $0.46-11.18$ & 0.132 \\
Pancreatic cancer & 3.14 & $0.69-20.22$ & 0.115 \\
Ascites & 8.39 & $1.29-375.4$ & 0.104 \\
Peritoneal carcinomatosis & 2.65 & $0.44-28.94$ & 0.305 \\
15mm LAMS & 2.45 & $0.14-14.09$ & 0.332 \\
Balloon dilation & 0.11 & $0.01-0.72$ & 0.059 \\
Learning curve, first 50\% & 1.30 & $0.37-15.49$ & 0.726 \\
\hline
\end{tabular}

Supplementary table 3. Learning curve assessment: comparison between first and second half of the EUS-GE cohorts.

\begin{tabular}{|c|c|c|c|c|c|}
\hline Propensity score-matched EUS-GE cohort: & $\begin{array}{l}\text { First } 50 \% \\
\quad(n=18)\end{array}$ & & $\begin{array}{l}\text { Second } 50 \% \\
\quad(n=19)\end{array}$ & & $P$-value \\
\hline Technical success, n (\%) & $17 / 18$ & $94.4 \%$ & $18 / 19$ & $94.7 \%$ & 1.000 \\
\hline Clinical success, n (\%) & $17 / 18$ & $94.4 \%$ & $17 / 19$ & $89.5 \%$ & 1.000 \\
\hline Per protocol clinical success, $\mathrm{n}(\%)$ & $17 / 17$ & $100 \%$ & $17 / 18$ & $94.4 \%$ & 1.000 \\
\hline Overall adverse events, $\mathrm{n}(\%)$ & 1 & $5.6 \%$ & 1 & $5.3 \%$ & 1.000 \\
\hline Median procedure duration, $\min (I Q R)$ & 51 & $(34-84)$ & 46 & $(40-83)$ & 0.719 \\
\hline Overall EUS-GE cohort: & $\begin{array}{c}\text { First } 50 \% \\
(n=38)\end{array}$ & & $\begin{array}{l}\text { Second } 50 \% \\
\quad(n=39)\end{array}$ & & $P$-value \\
\hline Technical success, $\mathrm{n}(\%)$ & $37 / 38$ & $97.4 \%$ & $36 / 39$ & $92.3 \%$ & 0.615 \\
\hline Clinical success, $n(\%)$ & $37 / 38$ & $97.4 \%$ & $34 / 39$ & $87.2 \%$ & 0.200 \\
\hline Per protocol clinical success, n (\%) & $37 / 37$ & $100 \%$ & $34 / 36$ & $94.4 \%$ & 0.240 \\
\hline Overall adverse events, n (\%) & 3 & $7.9 \%$ & 3 & $7.7 \%$ & 1.000 \\
\hline Median procedure duration, $\min (\mathrm{IQR})$ & 49 & $(36-78)$ & 52 & $(33-83)$ & 0.719 \\
\hline
\end{tabular}

Abbreviations: EUS-GE: Endoscopic ultrasound-guided gastroenterostomy, IQR: interquartile range. 


\section{Laparoscopic versus EUS-guided Gastroenterostomy for Gastric Outlet Obstruction: An International Multicentre Propensity Score-Matched Comparison.}

Michiel Bronswijk ${ }^{1,2}$, Giuseppe Vanella ${ }^{3}$, Hannah van Malenstein ${ }^{1}$, Wim Laleman ${ }^{1}$, Joris Jaekers ${ }^{4}$, Baki Topal ${ }^{4}$, Freek Daams ${ }^{5}$, Marc G. Besselink ${ }^{6}$, Paolo Giorgio Arcidiacono ${ }^{3}$, Rogier P. Voermans ${ }^{7}$, Paul Fockens $^{7,8}$, Alberto Larghi ${ }^{9}$, Roy LJ van Wanrooij ${ }^{8}$ and Schalk van der Merwe ${ }^{1}$

\section{Author affiliation:}

1. Department of Gastroenterology and Hepatology, University Hospitals Gasthuisberg, University of Leuven, Leuven, Belgium.

2. Department of Gastroenterology and Hepatology, Imelda General Hospital, Bonheiden, Belgium.

3. Pancreatobiliary Endoscopy and Endosonography Division, IRCCS San Raffaele Scientific Institute, Milan, Italy

4. Department of Visceral Surgery, University Hospitals Gasthuisberg, KU Leuven, Belgium.

5. Department of Surgery, Amsterdam UMC, Vrije Universiteit, Cancer Center Amsterdam, The Netherlands.

6. Department of Surgery, Amsterdam UMC, University of Amsterdam, Cancer Center Amsterdam, The Netherlands.

7. Department of Gastroenterology and Hepatology, Amsterdam UMC, University of Amsterdam, AGEM institute, Amsterdam, The Netherlands

8. Department of Gastroenterology and Hepatology, Amsterdam UMC, Vrije Universiteit Amsterdam, AGEM institute, Amsterdam, The Netherlands

9. Digestive Endoscopy Unit, Fondazione Policlinico Universitario A. Gemelli IRCCS, Università Cattolica del Sacro Cuore, Rome; CERTT, Center for Endoscopic Research Therapeutics and Training, Catholic University, Rome, Italy. 


\section{Abstract}

Background and aims:

In the management of gastric outlet obstruction (GOO), EUS-guided gastroenterostomy (EUS-GE) seems safe and more effective than enteral stenting. However, comparisons to laparoscopic gastroenterostomy (L-GE) are scarce. Our aim was to perform a propensity score-matched comparison between EUS-GE and L-GE.

\section{Methods:}

An international, multicentre retrospective analysis was performed of consecutive EUS-GE and L-GE procedures in 3 academic centers (Jan-2015 to May-2020), using propensity score-matching in order to minimize selection bias. A standard maximum propensity score difference of 0.1 was applied, also considering underlying disease and oncological staging.

Results:

Overall, 77 patients were treated with EUS-GE and 48 patients with L-GE. By means of propensity score-matching, 37 patients were allocated to both groups, resulting in 74 (1:1) matched patients.

Technical success was achieved in 35/37 EUS-GE-treated patients (94.6\%) vs. 100\% in the L-GE group ( $p=0.493$ ). Clinical success, defined as eating without vomiting or GOO Scoring System $\geq 2$, was achieved in $97.1 \%$ and $89.2 \%$ respectively ( $p=0.358)$. Median time to oral intake (1 (IQR 0.3-1.0) vs. 3 (IQR 1.0-5.0) days, $p<0.001$ ) and median hospital stay (4 (IQR 2-8) vs 8 (IQR 5.5-20) days, p<0.001) were significantly shorter in the EUS-GE group. Overall adverse events (AEs) $(2.7 \%$ vs. $27.0 \%$, $p=0.007)$ and severe AEs (0.0\% vs. $16.2 \%, p=0.025)$ were identified more frequently in the L-GE group.

Conclusion:

For patients with GOO, EUS-GE and L-GE showed almost identical technical and clinical success. However, reduced time to oral intake, shorter median hospital stay and lower rate of adverse events suggest that the EUS-guided approach might be preferable.

\section{Keywords}

Gastric outlet syndrome, laparoscopic gastrojejunostomy, endoscopic ultrasound, LAMS. 


\section{Introduction}

Gastric outlet obstruction (GOO), defined by a mechanical obstruction of the duodenum, pylorus or antrum, may result from various diseases. The underlying aetiology has shifted from mainly peptic ulcer disease in the past, to predominantly malignant causes at present ${ }^{1,2}$. In the most recent cohorts, GOO was caused by underlying malignancy in up to $85 \%$ of patients, the majority of which could be attributed to pancreatic cancer $3,4,5,6,7$. On the other hand, peptic ulcer disease and chronic pancreatitis are still the most prevalent causes of benign $\mathrm{GOO}^{8,9}$. Consequences of GOO, such as nausea, vomiting, anorexia, need for nasogastric tube decompression and subsequent loss of body mass, may further aggravate this complex clinical entity, increasing morbidity, reducing quality of life and significantly influencing tolerability and efficacy of oncological treatments ${ }^{10}$. Before the advent of endoscopic ultrasound-guided gastroenterostomy (EUS-GE), management of GOO mainly relied on surgical gastroenterostomy, the standard of care for many years ${ }^{11,12}$. Since the late nineties, placement of metal enteral stents was found to provide higher efficacy regarding early re-initiation of oral intake, as well as reduce hospital stay and major adverse events $(A E)^{11}$, albeit at the expense of more recurrent obstructive symptoms due to stent dysfunction ${ }^{13,14}$. Since 2012, EUS-GE has found its way from initial animal studies, into daily practice of tertiary centres ${ }^{15,16}$. This EUS-guided approach has become a minimally invasive alternative for patients with both benign or malignant $\mathrm{GOO}^{3,4}$ and demonstrated higher clinical success and lower need for re-interventions compared to enteral stenting ${ }^{5,17}$. There is still uncertainty regarding the place of EUS-GE in daily clinical practice, due to a lack of data comparing this technique to the current reference standard, laparoscopic gastroenterostomy (L-GE), in patients stratified according to potential confounders, such as oncological staging and pre-procedural fragility ${ }^{18-20}$.

Our aim was to perform a propensity score-matched comparison of EUS-GE to L-GE, using a large retrospective international multicentre cohort. 


\section{Methods}

\section{Patients and study design}

A retrospective analysis was performed of all consecutive L-GE and EUS-GE procedures performed for GOO at the Amsterdam University Medical Centers, location AMC and VUmc (the Netherlands), IRCCS San Raffaele Scientific Institute Milan (Italy) and University Hospitals Leuven (Belgium) between January 2015 and May 2020. For both procedures, identical variables were extracted from patients' electronical medical charts. Inclusion criteria consisted of: (1) symptomatic GOO, (2) endoscopic or radiological confirmation of benign or malignant gastro-duodenal stenosis and (3) treatment with EUS-GE or L-GE. Considering the surgical procedures, only strictly "pure" laparoscopic gastroenterostomies were eligible for inclusion, as to minimize confounding effects of adjunctive procedures, such as metastasectomy, hepatico-enterostomy or cholecystectomy, often performed simultaneously. Each patient gave his or her consent with regards to the procedure. This study was furthermore approved by the central Institutional Review Board (study identifier: s64254) at the University Hospitals Leuven and at each participating center.

\section{Endpoints}

Technical success was defined as the successful creation of a gastro-enteric anastomosis by use of the initially chosen technique (see below for technical considerations). If additional approaches or techniques had to be involved or additional self-expandable metal stents (SEMS) were needed, this was regarded as a technical failure.

Food intake grading was defined using the GOO Scoring System (GOOSS) ( $0=$ no intake, $1=$ liquid only, $2=$ soft solids, $3=$ almost full diet, $4=$ full diet) ${ }^{21,22}$; symptomatic GOO was defined as a GOOSS of 0 . In concordance with previous work ${ }^{7}$, clinical success was defined as eating without vomiting or a GOOSS of $\geq 2$ and was ascertained by evaluation of the electronic patient file, where this parameter is recorded as part of standard practice. Regarding safety, the ASGE lexicon for adverse events was used to stratify AE in mild, moderate, severe or fatal events ${ }^{23}$.

Secondary endpoints were: hospital stay, weight change after 2 months, gastroenterostomy dysfunction, distal obstruction rates, 'time to oral intake' and 'time to full diet' (GOOSS of 4). For EUS-GE procedural time was extracted from the endoscopic electronic reporting system. For L-GE, procedural time was retrieved by revision of the anaesthesiology report, exactly stating the beginning and the end of the procedure. Gastroenterostomy dysfunction was defined as recurrence of obstructive symptoms (GOOSS < 1) after former clinical success, with confirmation of recurrent GOO by endoscopy or imaging studies. Mechanical obstructions located downstream the GE site, without signs of EUS-GE or L-GE dysfunction, were annotated as 'distal obstructions', but not registered as GE dysfunction.

\section{Procedure: the Wireless EUS-gastroenterostomy Simplified Technique (WEST)}

All EUS-GE are performed under deep sedation with propofol or general anaesthesia, using a electrocautery-enhanced lumen apposing metal stent (LAMS) and the Wireless EUS-gastroenterostomy Simplified Technique (WEST) as previously described, under prophylactic antibiotic therapy (Video) ${ }^{24,25}$.

After a 7Fr nasobiliary catheter or enteral feeding tube is placed, through the gastric or duodenal stenosis into the jejunum, water is infused in the targeted loop of small bowel (Figure 1, upper left panel). Using a combination of fluoroscopy and EUSguided identification of the catheter (Figure 1, upper middle panel) the dilated jejunal or enteric loop is accessed using the biflanged electrocautery-enhanced LAMS (Hot-Axios; Boston Scientific, Marlborough, MA, USA). Following successful intraluminal access to the small bowel, the distal flange is deployed under endosonographic guidance; the device is then 
retracted (Figure 1, upper right panel), favouring apposition of the gastric and enteric wall and allowing the opening of the proximal flange inside the endoscope channel (Figure 1, lower left panel); the device is finally pushed outside the working channel together with a careful scope retraction, resulting in deployment of the proximal flange into the gastric lumen. Afterwards, successful deployment is confirmed by either EUS (Figure 1, lower left panel), direct endoscopic visualisation (Figure 2) or, if needed, combined with fluoroscopy and contrast injection (Figure 1, lower middle panel).

\section{Procedure: Laparoscopic gastroenterostomy:}

Following CO2-insufflation (12-15 mmHg intra-abdominal pressure) by a Veress needle or by open introduction, 4-5 trocars are introduced: one camera port around the umbilicus and 3 to 4 trocars at different positions in the upper abdomen. Next, Treitz' ligament is identified and two electrocautery incisions are made, one in the dorsal or anterior gastric wall and one in the jejunum. An anterior, dorsal latero-lateral or side-to-side isoperistaltic gastroenteric anastomosis is constructed. The exact location of the gastroenteric anastomosis, with regards to Treitz' ligament, varies from $30 \mathrm{~cm}$ to $60 \mathrm{~cm}$. During the surgical approach a 60mm blue Echelon stapler (Johnson \& Johnson, New Brunswick, New Jersey, USA) or 60mm Endo GIA universal stapler (Medtronic Ltd., Dublin, Ireland) is used depending on the preference of the surgeon, with additional staplers and sutures covering the staple line as needed. During construction of the gastroenteric anastomosis, a 36 French nasogastric tube is temporarily placed through the defect in the majority of cases, in an effort to maintain and confirm patency while stapling or suturing. Anti-traction sutures are used when appropriate. If not in place before the surgical procedure, a nasogastric tube is inserted afterwards and the patient remained on nil per mouth. In this study we only included "pure" L-GE for comparison to EUS-GJ and excluded procedures were adjunctive treatments, such as cholecystectomy, hepaticojejunostomy or metastasectomy were performed. All surgical procedures were performed by gastrointestinal surgeons with extensive experience in laparoscopic gastrointestinal surgery, operating in high-volume academic centres.

\section{Statistical analysis}

Categorical variables were reported as frequencies (\%) and Fisher's test was used to compare these variables. Continuous variables were reported as medians and interquartile range (IQR) or means \pm standard deviation (SD). Student's $t$ test and Mann-Whitney $U$ test were used for comparing continuous variables as appropriate. Differences in outcomes are shown as odds ratio (OR) and 95\% confidence interval (CI). Kaplan-Meier curves were used for overall post-procedural survival analysis, whereas the log-rank test was used for corresponding comparisons. Furthermore, learning curve effect was evaluated by comparing the initial $50 \%$ of procedures with the second half of EUS-GE procedures in each centre. A multiple logistic regression was performed to identify predictors of clinical failure: age, gender, pancreatic cancer, presence of ascites or peritoneal carcinomatosis, use of $15 \mathrm{~mm}$ LAMS and balloon dilation, as well as learning curve were used as variables. P-values $<0.05$ were considered statistically significant.

A propensity score-matched analysis was performed in an effort to minimize selection bias. Age, sex, underlying disease, corresponding disease stage, presence of ascites and presence of peritoneal carcinomatosis were used as variables. The aforementioned variables were selected based on: (1) analysis of previous literature and (2) significant differences between the two groups after univariate analysis. In four previously published studies, which performed a multivariate analysis, peritoneal carcinomatosis and ascites were identified as factors associated with clinical and technical failure respectively3,4,7,26 A standard maximum propensity score difference of 0.1 was admitted for matching.

SPSS version 26.0 (IBM, Chicago, IL, USA) was used for matching and statistical analysis, whereas Graphpad Prism version 9.0.0 for Windows (Graphpad Software, San Diego, CA, USA) was used for the Kaplan-Meier curves and survival analysis. 


\section{Results}

Overall, we identified 126 patients, one of whom was excluded due to missing data, resulting in a total of 77 patients (62\%) who underwent EUS-GE and 48 patients (38\%) undergoing L-GE.

Baseline characteristics are shown in Table 1. Ascites (22.1\% vs. 4.2\%, p=0.009) and pancreatic cancer-induced GOO (48.1\% vs. $29.2 \%, p=0.037$ ) were significantly more frequent in the EUS-GE group, whereas underlying benign disease was identified significantly less (3.9\% vs. 14.6\%, p=0.044) when compared to L-GE-treated patients (Table 1). Technical success (94.8\% (95\% Cl: $87.0-98.4)$ vs. $100 \%$ (95\% Cl: 91.2-100.0), p=0.297) was similar when comparing both groups, while a trend towards higher per-protocol clinical success amongst EUS-GE-treated patients was observed (97.3\% (95\% Cl: 90.0-99.8) vs. 87.5\% (95\% Cl: 74.9-94.5), OR 5.07 (95\% Cl: 0.98-26.28), p=0.057) (Table 2).

Overall, $15 \mathrm{~mm}$ and $20 \mathrm{~mm}$ LAMS were utilized in 11 and 64 patients respectively, with higher clinical success rates $(81.8 \%$ (95\% Cl: $51.2-96.0)$ vs. $100 \%(95 \% \mathrm{Cl}: 93.0-100.0)$ respectively, $\mathrm{p}=0.021)$ and a trend towards shorter median hospital stay (5 days (2.8-11) vs. 4 days (2.0-11), p=0.054) when $20 \mathrm{~mm}$ LAMS were utilized. Intraprocedural balloon dilation of the central part of the LAMS immediately after its placement was performed in $26 \%$ of patients and did not affect efficacy outcomes (Supplementary Table 1). Although two primary clinical failures occurred in patients in whom 15mm LAMS were used without dilation, multivariate analysis did not identify any significant independent risk factors for clinical failure (Supplementary Table 2). Overall AEs (6.5\% (95\% Cl: 2.5-14.7) vs 31.3\% (95\% Cl: 19.9-45.4), OR 0.15 (95\% Cl: 0.05-0.46), p<0.001), and severe AEs (2.6\% (95\% Cl: $0.2-9.5)$ vs $18.8 \%(95 \% \mathrm{Cl}: 10.0-32.2), \mathrm{OR} 0.12(95 \% \mathrm{Cl}: 0.03-0.59), \mathrm{p}=0.007)$ occurred significantly less frequently in EUS-GE versus L-GE treated patients (Table 2).

\section{Propensity score-matched analysis}

By means of propensity score matching, 37 patients were allocated to each group, resulting in a total of 74 (1:1) matched patients (Figure 3). The propensity score-matched cohort revealed an overall mean age of $66.5 \pm 11.8$ years, $44.6 \%$ were female, $36.5 \%$ had underlying pancreatic cancer, with $31.1 \%$ and $6.8 \%$ of patients exhibiting peritoneal carcinomatosis and ascites. Underlying benign disease was present in four (5.4\%) patients. Baseline comparisons between EUS-GE and L-GEtreated patients are shown in Table 3. No significant differences between both study groups were identified.

\section{Efficacy}

In the propensity score-matched EUS-GE group, two technical failures occurred, due to inability to advance the nasobiliary catheter through the stenosis. This resulted in a technical success rate of 94.6\% (35 out of 37 patients, 95\% Cl: 81.4-99.4), compared to $100 \%$ (95\% Cl: 88.8-100.0), p=0.493) amongst L-GE-treated patients (Table 4). Clinical success rates by means of intention to treat analysis (91.9\% (95\% Cl: 78.0-97.9) vs. 89.2\% (95\% Cl: 74.7-96.3), p=1.000), as well as per-protocol analysis were comparable (97.1\% (95\% Cl: 84.2-100.0) vs. 89.2\% (95\% Cl: 74.7-96.3), p=0.358), with primary non-functional surgical gastroenterostomy in three L-GE-patients. Procedure time (46 minutes (IQR 37.5-80.0) vs. 85 minutes (73.0-110), p<0.001), median time to oral intake (1 day (IQR 0.3-1.0) vs. 3 days (IQR 1.0-5.0), p<0.001) and median time to full diet (2 days (IQR 1.03.8) vs. 9 days (IQR 4.0-23), p<0.001) were significantly shorter in the EUS-GE group.

After a median follow-up of 77 days (IQR 27-160) in the EUS group and 123 days in the surgical group (IQR 32-262), gastroenterostomy dysfunction rates (none in both groups) did not differ. With two-months' weight change available in $51.4 \%$ and $56.8 \%$ of patients treated with EUS-GE and L-GE respectively, no significant differences were detected (-0.3kg (IQR -2.41.1) vs. Okg (IQR -3.0-0.7), $p=0.159)$. 


\section{Safety and postoperative outcomes}

The overall number of AEs in the propensity score-matched cohort was lower amongst EUS-GE treated patients (2.7\% (95\% Cl: $0.01-15.1)$ vs. $27 \%$ (95\% Cl: 15.2-43.1), OR 0.07 (95\% Cl: 0.01-0.62), p=0.007) (Table 4). Most AEs in the L-GE group (6 out of 10 ) were severe, mainly consisting of anastomotic leaks $(n=4,10.8 \%)$ or bleeding $(n=2,5.4 \%)$, necessitating surgical reintervention in three patients (8.1\%), while no severe AEs were registered among EUS-GE treated patients $(0.0 \%(95 \% \mathrm{Cl}$ : $0.0-11.2$ ) vs. $16.2 \%$ (95\% Cl: $7.3-31.5)$, OR 0.07 (95\% Cl: $0.00-1.19), p=0.025)$. Mild (2.7\% in each group) and moderate $A E$ rates $(0.0 \%(95 \% \mathrm{Cl}: 0.0-11.2)$ vs. $8.1 \%(95 \% \mathrm{Cl}: 2.1-22.0), \mathrm{p}=0.240)$ were similar in both groups. In two L-GE patients, endoscopic reinterventions was deemed necessary in the context of postoperative bleeding and placement of a transanastomotic stent to treat a dysfunctional surgical anastomosis.

A significantly shorter median hospital stay (4 days (IQR 2.0-8.0) vs. 8 days (IQR 5.5-20), p<0.001) was observed amongst EUSGE-treated patients. Survival analysis did not reveal a significant difference in post-procedural survival in the matched cohort, nor in the overall cohort (Figure 4).

\section{Learning Curve Assessment}

When comparing the first to the second half of both the overall and propensity score-matched EUS-GE cohorts (Supplementary Table 3), no significant differences were found in terms of safety and efficacy. 


\section{Discussion}

In the current analysis, we performed the first propensity score-matched comparison between EUS-GE and L-GE. We found that EUS-GE achieved similar technical and clinical success, with significantly lower overall and severe AEs, faster resumption of oral intake and earlier discharge compared to L-GE. Whilst the EUS-GE technical and clinical success rate was in line with previously published studies $3-9,27$, we observed that technical success depended mainly on the ability to pass a nasobiliary catheter through the stenosis. Furthermore, amongst all technical variables, only LAMS calibre might have some influence on clinical outcome, although this was not confirmed by multivariate analysis.

The paucity of published comparative data makes it difficult to identify a well-defined place for EUS-GE in the management of patients with GOO, especially compared to the more established techniques such as surgery and enteral stenting. Only a single large multicentre retrospective study comparing open gastroenterostomy to EUS-GE has been published so far ${ }^{7}$. The authors reported lower AEs compared to the open surgical approach, at the cost of a lower, but not statistically significant, technical success rate. Surprisingly, length of hospital stay amongst both groups was similar. One can speculate whether this was related to the limited experience available at the time, which may have influenced clinical decisions regarding timing of discharge and general post-EUS-GE management. In our cohort, most patients were discharged after a median of 4 days (IQR 2.0-8.0) compared to 8 days (IQR 5.5-20) in the L-GE group, which may an impact on health care costs and quality of life. We chose to compare our EUS-GE cohort with laparoscopic surgery, as it has proven superior to open surgery, in and outside the context of gastroenterostomy, showing lower morbidity and earlier recovery compared to open surgery, making the L-GE approach the most desired comparator ${ }^{18}$. One previous multicentre retrospective analysis evaluated the efficacy and safety of EUS-GE compared to L-GE, this study only included 25 patients undergoing EUS-GE and did not correct for potential confounding factors or bias ${ }^{19}$. The authors demonstrated increased safety and lower costs using EUS-GE, while retaining similar efficacy to L-GE, even if they detected a non-significant difference in technical success in favour of L-GE ${ }^{19}$ The surgical adverse event rate observed in our study (31.3\%), was similar to those reported by Perez-Miranda, et al $(41 \%)^{19}$ and Kashab, et al $(25 \%)^{7}$, suggesting that the superior safety outcomes of EUS-GE observed in our analysis were not due to inferior performance of the surgical comparator group. Furthermore, when comparing surgical adverse events rates in historical cohorts of surgical palliative gastroenterostomy, similar ${ }^{28-30}$ or higher ${ }^{31-33}$ adverse event rates were seen when compared to the current analysis.

When considering previous comparisons of EUS-GE with enteral stenting, limited evidence suggests that the latter is associated with a lower rate of clinical success and higher rates of stent failure requiring re-intervention 5,17 . These findings have been confirmed in studies comparing enteral stenting with surgical gastroenterostomy, suggesting that enteral stenting should be considered in the context of very limited life expectancy only13,34.

There are some issues when comparing previous EUS-GE data with our current analysis. First, several techniques have been described for performing EUS-GE, which include the endoscopic ultrasonography-guided double-balloon-occluded gastrojejunostomy bypass (EPASS)-technique, natural orifice transluminal endoscopic surgery (NOTES), rendez-vous methods including balloon-assisted gastroenterostomy, and the direct or 'free-hand'-techniques ${ }^{7,35-37}$. These techniques have been interchangeably used throughout several studies $3,4,7,8,19$, complicating reliable direct comparisons of results. Secondly, several previous papers have published overlapping study cohorts, rendering data interpretation somewhat complicated 4,19,20,35,38. Third, the use of different LAMS with a limited number of studies also including cases where Niti-S Spaxus LAMS (Taewoong Medical Co. Ltd., Ilsan, Korea) were used instead of the Hot-Axios ${ }^{3,7,26}$. Regarding different EUS-GE approaches, a comparative study has shown that the direct method achieves similar technical and clinical success, with a similar safety profile when 
compared to balloon-assisted EUS-GE ${ }^{3}$. However, in the context of the direct method, procedure time was more than twice shorter (35.7 vs. 89.9min, $\mathrm{p}<0.001$ ), thus suggesting the direct technique as the preferred method. In all of our patients, only the Wireless EUS-gastroenterostomy Simplified Technique (WEST) was used, indicating that there is no need for a 19G 'finder'-needle, as has been described in the direct technique, or a guidewire and balloon in order to perform EUS-GE safely and effectively ${ }^{3,24,25}$. We recommend against inflating the targeted loop of small bowel by water or contrast injection using a 19-gauge needle, such as in the setting of EUS-directed transenteric ERCP (EDEE) ${ }^{39}$, an approach which may carry a higher theoretical risk of puncturing a more distal enteric loop or even the colon. We do recommend using the most straightforward technique available, in an effort to reduce the number of additional accessories requiring exchange, which in our opinion carries an increased risk of adverse events by complicating positioning, visualisation and, as time passes, reduction in small bowel distention.

While the usefulness of EUS-GE in malignant disease has been reported in various studies, the evidence of this procedure in benign disease has been increasing since only recently5,7,17. Doubts concerning LAMS patency and long-term results have led to restricted use in benign diseases. In 2020, James et al. published their series on EUS-GE in benign disease, revealing that surgery was averted in $83.3 \%$ of patients and regression of the benign stricture allowed for LAMS removal in the majority of patients over time ${ }^{9}$. Together with long-term follow-up data published in $2019^{6}$, which showed a $15 \%$ recurrence rate after a median follow-up time of 169 (malignant disease) and 319.5 days (benign disease), we can conclude that especially in patients with malignant disease GOO, recurrence is an issue. In our current propensity score-matched analysis gastroenterostomy dysfunction did not occur at all, although two cases of distal enteric obstruction, due to metastatic peritoneal disease, were identified in the EUS-GE group, compared to one in the L-GE-group.

In comparison to various other studies, where there was no mention of the incidence of ascites $3,4,6-9,17,19,21 \%$ of our patients underwent EUS-GE despite the presence of ascites, without any significant related AEs. Although ascites has been regarded as a strict contraindication for EUS-GE, these results, together with a retrospective analysis in $2019^{5}$, suggest that patients with mild or localized ascites can be considered for EUS-GE without risking leakage or subsequent peritonitis, provided that there is no tense ascites and that the LAMS trajectory is not compromised due to fluid interference.

\section{Limitations and strengths:}

Several limitations of the current study should be addressed. First, the retrospective nature of this analysis might have inadvertently introduced some bias. Secondly, due to the study design, a certain degree of missing data was identified, most especially in the context of body weight evolution. Third, generalizability of our data might be an issue, as all endoscopists were highly trained and operating in high-volume settings.

We have tried to address some of these limitations in our study design. We included a propensity score-matched design, correcting for the selected variables to limit selection bias. These specific variables were chosen as they were differently distributed amongst the two treatment groups, whilst potentially influencing technical and clinical success, as well as 'time to oral intake', 'time to full diet', overall survival and gastroenterostomy dysfunction rates. One of the disadvantages of propensity score-matching, is the dependence on the matching criteria. We therefore included various variables as to provide a stringent matching process. To reduce larger treatment effects and higher degrees of bias of single centre studies, we involved three different tertiary referral centres recruiting similar patients and all performing EUS-GE using the WEST approach $^{24,25}$. Finally, with 77 patients in whom EUS-GE was performed, our study is one of the largest published original cohorts of EUS-GE, and the largest study to date to compare EUS-GE with L-GE. 
In conclusion, this study suggests that in patients with gastric outlet syndrome, EUS-GE and L-GE provide almost identical technical and clinical success rates. Lower time to oral intake, shorter hospital stay and a lower rate of adverse events prudentially suggest that EUS-GE should be the preferred approach in patients with GOO. While awaiting high-quality prospective confirmation, these findings should guide gastroenterologists, oncologists and surgeons in considering EUS-GE for treating GOO, especially in the setting of malignancy, where patients will benefit from the least invasive technique with the highest expected efficacy.

\section{Acknowledgements}

None

\section{References}

1. McCarty TR, Garg R, Thompson CC, Rustagi T. Efficacy and safety of EUS-guided gastroenterostomy for benign and malignant gastric outlet obstruction: a systematic review and meta-analysis. Endosc Int Open 2019;7:E1474-E1482.

2. Shone DN, Nikoomanesh P, Smith-Meek MM et al. Malignancy is the most common cause of gastric outlet obstruction in the era of H2 blockers. Am J Gastroenterol 1995;90:1769-1770

3. Chen YI, Kunda R, Storm AC, et al. EUS-guided gastroenterostomy: a multicenter study comparing the direct and balloonassisted techniques. Gastrointest Endosc 2018;87:1215-21.

4. Tyberg A, Perez-Miranda M, Sanchez-Ocaña R, et al. Endoscopic ultrasound-guided gastrojejunostomy with a lumenapposing metal stent: a multicenter, international experience. Endosc Int Open 2016;4:E276-81.

5. Ge PS, Young JY, Dong W, et al. EUS-guided gastroenterostomy versus enteral stent placement for palliation of malignant gastric outlet obstruction. Surg Endosc 2019;33:3404-11.

6. Kerdsirichairat T, Irani S, Yang J, et al. Durability and long-term outcomes of direct EUS-guided gastroenterostomy using lumen-apposing metal stents for gastric outlet obstruction. Endosc Int Open 2019;7:E144-E150.

7. Khashab MA, Bukhari M, Baron TH, et al. International multicenter comparative trial of endoscopic ultrasonographyguided gastroenterostomy versus surgical gastrojejunostomy for the treatment of malignant gastric outlet obstruction. Endosc Int Open 2017;5:E275-E281

8. Chen YI, James TW, Agarwal A, et al. EUS-guided gastroenterostomy in management of benign gastric outlet obstruction. Endosc Int Open 2018;6:E363-E368.

9. James TW, Greenberg S, Grimm IS, Baron TH. EUS-guided gastroenteric anastomosis as a bridge to definitive treatment in benign gastric outlet obstruction. Gastrointest Endosc 2020;91:537-542.

10. Klute KA, Brouwer J, Jhawer M, et al. Chemotherapy dose intensity predicted by baseline nutrition assessment in gastrointestinal malignancies: A multicentre analysis. Eur J Cancer 2016;63:189-200. 
11. Ly J, O'Grady G, Mittal A, et al. A systematic review of methods to palliate malignant gastric outlet obstruction. Surg Endosc 2010;24:290-297.

12. Wilson RG, Varma JS. Laparoscopic gastroenterostomy for malignant duodenal obstruction. Br J Surg 1992;79:1348.

13. Jeurnink SM, Van Eijck CHJ, Steyerberg EW, et al. Stent versus gastrojejunostomy for the palliation of gastric outlet obstruction: a systematic review. BMC Gastroenterol 2007;7:1-10.

14. Khashab M, Alawad AS, Shin EJ, et al. Enteral stenting versus gastrojejunostomy for palliation of malignant gastric outlet obstruction. Surg Endosc 2013;27:2068-2075.

15. Binmoeller KF, Shah JN. Endoscopic ultrasound-guided gastroenterostomy using novel tools designed for transluminal therapy: a porcinestudy. Endoscopy 2012;44:499-503

16. Itoi T, Ishii K, Tanaka R, et al. Current status and perspective of endoscopicultrasonography-guided gastrojejunostomy: endoscopic ultrasonography-guided double-balloon-occluded gastrojejunostomy (with videos). J Hepatobiliary Pancreat Sci 2015;22:3-11

17. Chen YI, Itoi T, Baron TH, et al. EUS-guided Gastroenterostomy is comparable to enteral stenting with fewer reinterventions in malignant gastric outlet obstruction. Surg Endosc 2017;31:2946-2952.

18. Al-Rashedy M, Dadibhai M, Shareif A, et al. Laparoscopic gastric bypass for gastric outlet obstruction is associated with smoother, faster recovery and shorter hospital stay compared with open surgery. J Hepatobiliary Pancreat Surg $2005 ; 12: 474-478$.

19. Perez-Miranda M, Tyberg A, Poletto D, et al. EUS-guided gastrojejunostomy versus laparoscopic gastrojejunostomy: an international collaborative study. J Clin Gastroenterol 2017;51:896-899.

20. Khashab MA, Kumbhari V, Grimm IS, et al. EUS-guided gastroenterostomy: the first U.S. clinical experience (with video). Gastrointest Endosc 2015;82:932-938.

21. Brewer Gutierrez OI, Nieto J, Irani S, et al. Double endoscopic bypass for gastric outlet obstruction and biliary obstruction. Endosc Int Open 2017;5:E893-E899.

22. Adler DG, Baron TH. Endoscopic palliation of malignant gastric outlet obstruction using self-expanding metal stents: experience in 36 patients. Am J Gastroenterol 2002;97:72-78.

23. Cotton PB, Eisen GM, Aabakken L, et al. A lexicon for endoscopic adverse events: report of an ASGE workshop. Gastrointest Endosc 2010;71:446-454.

24. Bronswijk M, Fransen L, Vanella G, et al. Successful treatment of superior mesenteric artery syndrome by endoscopic ultrasound-guided gastrojejunostomy [published online ahead of print, 2020 Jun 19]. Endoscopy 2020;10.1055/a-11903228.

25. Bronswijk M, Vanella G, Petrone MC, et al. EUS-guided gastroenterostomy: Less is more! The wireless EUS-guided gastroenterostomy simplified technique. VideoGIE 2020;5:442. 
26. Wannhoff A, Ruh N, Meier B, et al. Endoscopic gastrointestinal anastomoses with lumen-apposing metal stents: predictors of technical success [published online ahead of print, 2020 May 1]. Surg Endosc 2020;10.1007/s00464-02007594-5.

27. Antonelli G, Kovacevic B, Karstensen JG, et al. Endoscopic ultrasound-guided gastro-enteric anastomosis: A systematic review and meta-analysis. Dig Liver Dis 2020;52:1294-1301.

28. Bednarsch J, Czigany Z, Heise D, et al. Influence of peritoneal carcinomatosis on perioperative outcome in palliative gastric bypass for malignant gastric outlet obstruction - a retrospective cohort study. World J Surg Oncol 2020;18:25.

29. Denley SM, Moug SJ, Carter CR, McKay CJ. The outcome of laparoscopic gastrojejunostomy in malignant gastric outlet obstruction. Int J Gastrointest Cancer 2005;35:165-169.

30. Kazanjian KK, Reber HA, Hines OJ. Laparoscopic gastrojejunostomy for gastric outlet obstruction in pancreatic cancer. Am Surg 2004;70:910-913.

31. Jeurnink SM, Steyerberg EW, Hof Gv, et al. Gastrojejunostomy versus stent placement in patients with malignant gastric outlet obstruction: a comparison in 95 patients. J Surg Oncol 2007;96:389-396.

32. Zhang LP, Tabrizian P, Nguyen S, et al. Laparoscopic gastrojejunostomy for the treatment of gastric outlet obstruction. JSLS 2011;15:169-173.

33. Kuriansky J, Sáenz A, Astudillo E, et al. Simultaneous laparoscopic biliary and retrocolic gastric bypass in patients with unresectable carcinoma of the pancreas. Surg Endosc 2000;14:179-181.

34. Jeurnink SM, Steyerberg EW, van Hooft JE, et al. Surgical gastrojejunostomy or endoscopic stent placement for the palliation of malignant gastric outlet obstruction (SUSTENT study): a multicenter randomized trial. Gastrointest Endosc 2010;71:490-9.

35. Itoi T, Ishii K, Ikeuchi N, et al. Prospective evaluation of endoscopic ultrasonography-guided double-balloon-occluded gastrojejunostomy bypass (EPASS) for malignant gastric outlet obstruction. Gut 2016;65:193-195.

36. Irani S, Baron TH, Itoi T, Khashab MA. Endoscopic gastroenterostomy: techniques and review. Curr Opin Gastroenterol 2017;33(5):320-329.

37. Barthet M, Binmoeller KF, Vanbiervliet G, et al. Natural orifice transluminal endoscopic surgery gastroenterostomy with a biflanged lumenapposing stent: first clinical experience (with videos). Gastrointest Endosc 2015;81:215-218.

38. Khashab MA, Baron TH, Binmoeller KF, et al. EUS-guided gastroenterostomy: a new promising technique in evolution. Gastrointest Endosc 2015;81:1234-1236.

39. Ichkhanian Y, Yang J, James TW, et al. EUS-directed transenteric ERCP in non-Roux-en-Y gastric bypass surgical anatomy patients (with video). Gastrointest Endosc 2020;91:1188-1194. 


\section{Figure legend}

Figure 1. Stepwise approach to the Wireless EUS-gastroenterostomy Simplified Technique (WEST).

Upper left: Fluoroscopic image, placement of a 7Fr nasobiliary catheter or enteral feeding tube through the gastric or duodenal stenosis into the jejunum. Confirmation of the distal position by contrast opacification.

Upper middle: Endoscopic ultrasound image, showing distention of the targeted loop of small bowel after infusion of water. Upper right: Using a combination of fluoroscopy and EUS-guided identification of the catheter, the dilated jejunal or enteric loop is accessed using the biflanged electrocautery-enhanced LAMS, after which the distal flange is deployed under endosonographic guidance. The device is then retracted onto the gastric wall.

Lower left: Endoscopic ultrasound image, after compressing the gastric and enteric wall, as well as deployment of the proximal flange inside the endoscope channel, the device is finally pushed outside the working channel together with a careful scope retraction, resulting in deployment of the proximal flange into the gastric lumen.

Lower middle: Fluoroscopic evaluation after LAMS release, showing the successful deployment of the LAMS between the stomach and small bowel.

Lower right: Endoscopic view, after recannulation with a diagnostic catheter.

Figure 2. Direct endoscopic visualisation of successful gastroenterostomy.

Figure 3. Study flowchart.

Figure 4. Post-procedural survival analysis. 


\section{Tables}

Table 1. Overall baseline characteristics.

\begin{tabular}{|c|c|c|c|c|c|}
\hline & $\begin{array}{c}\text { EUS-GE } \\
(n=77)\end{array}$ & & $\begin{array}{c}\text { L-GE } \\
(n=48)\end{array}$ & & $P$ value \\
\hline \multicolumn{6}{|l|}{ Variable } \\
\hline Age (years), mean \pm SD & 65 & \pm 12.3 & 66 & \pm 11.6 & 0.478 \\
\hline Female, $\mathrm{n}(\%)$ & 36 & $(46.8 \%)$ & 19 & (39.6\%) & 0.432 \\
\hline Median follow up duration, days (IQR) & 76 & $(36-136)$ & 122 & $(35-274)$ & 0.057 \\
\hline \multicolumn{6}{|l|}{ Primary disease } \\
\hline Pancreatic cancer & 37 & $(48.1 \%)$ & 14 & $(29.2 \%)$ & 0.037 \\
\hline Biliary/gallbladder cancer & 9 & $(11.7 \%)$ & 2 & $(4.2 \%)$ & 0.149 \\
\hline Gastric cancer & 7 & $(9.1 \%)$ & 5 & $(10.4 \%)$ & 0.807 \\
\hline Duodenal cancer & 11 & $(14.3 \%)$ & 10 & $(20.8 \%)$ & 0.341 \\
\hline Breast cancer & 2 & $(2.6 \%)$ & 2 & $(4.2 \%)$ & 0.463 \\
\hline Colorectal cancer & 2 & $(2.6 \%)$ & 1 & $(2.1 \%)$ & 1.000 \\
\hline NET & 1 & $(1.3 \%)$ & 0 & $(0.0 \%)$ & 0.384 \\
\hline Ampullary cancer & 0 & $(0.0 \%)$ & 1 & $(2.1 \%)$ & 0.384 \\
\hline NSCLC & 3 & $(3.9 \%)$ & 1 & $(2.1 \%)$ & 1.000 \\
\hline Benign disease & 3 & $(3.9 \%)$ & 7 & $(14.6 \%)$ & 0.044 \\
\hline \multicolumn{6}{|l|}{ Disease stage } \\
\hline Local invasion & 25 & $(32.5 \%)$ & 13 & $(27.1 \%)$ & 0.555 \\
\hline Liver metastases & 8 & $(10.4 \%)$ & 9 & $(18.8 \%)$ & 0.193 \\
\hline Peritoneal metastastases & 8 & $(10.4 \%)$ & 10 & $(20.8 \%)$ & 0.122 \\
\hline Diffuse metastatic & 19 & $(24.7 \%)$ & 9 & $(18.8 \%)$ & 0.512 \\
\hline \multicolumn{6}{|l|}{ Disease manifestations } \\
\hline Ascites & 17 & $(22.1 \%)$ & 2 & $(4.2 \%)$ & 0.009 \\
\hline Peritoneal carcinomatosis & 20 & $(26.0 \%)$ & 16 & (33.3\%) & 0.420 \\
\hline
\end{tabular}


Table 2. Overall outcomes.

1

\begin{tabular}{|c|c|c|c|c|c|}
\hline & $\begin{array}{c}\text { EUS-GE } \\
(n=77)\end{array}$ & & $\begin{array}{c}\text { L-GE } \\
(n=48)\end{array}$ & & OR $(95 \% \mathrm{Cl}), P$ value \\
\hline \multicolumn{6}{|l|}{ Efficacy } \\
\hline Technical success, n (\%) & 73 & $(94.8 \%)$ & 48 & $(100 \%)$ & $0.17(0.01-3.20), 0.297$ \\
\hline Clinical success, n (\%) & 71 & $(92.2 \%)$ & 42 & $(87.5 \%)$ & $1.69(0.51-5.58), 0.534$ \\
\hline Per protocol clinical success, n (\%) & 71 & $(97.3 \%)$ & 42 & $(87.5 \%)$ & $5.07(0.98-26.28), 0.057$ \\
\hline Median time to oral intake, days (IQR) & 1 & $(0-1)$ & 3 & $(1-5)$ & $<0.001$ \\
\hline Full diet tolerability, n (\%) & 32 & $(41.6 \%)$ & 19 & (39.6\%) & $1.16(0.56-2.44), 0.854$ \\
\hline Median time to full diet, days (IQR) & 2 & $(1-4)$ & 8 & $(4-21)$ & $<0.001$ \\
\hline Gastroenterostomy dysfunction, n (\%) & 1 & $(1.3 \%)$ & 0 & $(0.0 \%)$ & $1.90(0.08-47.64), 1.000$ \\
\hline Median time to dysfunction, days (IQR) & 243 & N/A & N/A & N/A & N/A \\
\hline Distal obstruction, n (\%) & 8 & $(10.4 \%)$ & 1 & $(1.3 \%)$ & $5.45(0.66-45.02), 0.151$ \\
\hline Median time to distal obstruction, days (IQR) & 34 & $(18-138)$ & 13 & N/A & $\mathrm{N} / \mathrm{A}$ \\
\hline \multicolumn{6}{|l|}{ Safety } \\
\hline Overall adverse events, n (\%) & 5 & $(6.5 \%)$ & 15 & $(31.3 \%)$ & $0.15(0.05-0.46),<0.001$ \\
\hline Mild, $n(\%)$ & 2 & $(2.6 \%)$ & 1 & $(2.1 \%)$ & $1.25(0.11-14.21), 1.000$ \\
\hline Post-procedural fever, $\mathrm{n}(\%)$ & 2 & $(2.6 \%)$ & 0 & $(0.0 \%)$ & $3.21(0.15-68.35), 0.523$ \\
\hline Moderate, $\mathrm{n}(\%)$ & 1 & $(1.3 \%)$ & 5 & $(10.4 \%)$ & $0.11(0.01-1.00), 0.106$ \\
\hline Sepsis, n (\%) & 1 & $(1.3 \%)$ & 0 & $(0.0 \%)$ & $1.90(0.08-47.64), 1.000$ \\
\hline \multirow[t]{2}{*}{ Need for re-endoscopy, n (\%) } & 0 & $(0.0 \%)$ & 4 & $(8.3 \%)$ & $0.06(0.00-1.21), 0.020$ \\
\hline & 2 & $(2.6 \%)$ & 9 & $(18.8 \%)$ & $0.12(0.03-0.59), 0.007$ \\
\hline Intra-peritoneal LAMS deployment, n (\%) & 2 & $(2.6 \%)$ & N/A & $N / A$ & $N / A$ \\
\hline Anastomotic leak, n (\%) & N/A & N/A & 3 & $(6.3 \%)$ & $N / A$ \\
\hline Anastomotic bleeding, $\mathrm{n}(\%)$ & N/A & N/A & 2 & $(4.2 \%)$ & $\mathrm{N} / \mathrm{A}$ \\
\hline Surgical re-intervention, n (\%) & N/A & N/A & 3 & $(6.3 \%)$ & N/A \\
\hline Fatal, $\mathrm{n}(\%)$ & 0 & $(0.0 \%)$ & 0 & $(0.0 \%)$ & 1.000 \\
\hline \multicolumn{6}{|l|}{ Other } \\
\hline Median procedure duration, min (IQR) & 51 & $(36-79.8)$ & 95 & $(75-118)$ & $<0.001$ \\
\hline Median hospital stay, days (IQR) & 4 & $(2-10.5)$ & 8 & $(5-20)$ & $<0.001$ \\
\hline Median weight change after 2 months, kg (IQR) & -1 & $(-4.0-1.1)$ & $-0,4$ & $(-4.2-0.8)$ & 0.390 \\
\hline Median post-procedural survival, days (IQR) & 103 & $(44-252)$ & 147 & $(68-335)$ & 0.246 \\
\hline
\end{tabular}

Abbreviations: Cl: confidence interval, EUS-GE: Endoscopic ultrasound-guided gastroenterostomy, IQR: interquartile range, LGE: laparoscopic gastroenterostomy, N/A: not applicable, OR: odds ratio. 
Table 3. Matched cohort: baseline characteristics.

\begin{tabular}{|c|c|c|c|c|c|}
\hline & $\begin{array}{c}\text { EUS-GE } \\
(n=37)\end{array}$ & & $\begin{array}{c}\text { L-GE } \\
(n=37)\end{array}$ & & $P$ value \\
\hline \multicolumn{6}{|l|}{ Variable } \\
\hline Age (years), mean \pm SD & 66.5 & \pm 12.5 & 66.4 & \pm 11.1 & 0.954 \\
\hline Female, $\mathrm{n}(\%)$ & 18 & $(48.7 \%)$ & 15 & $(40.5 \%)$ & 0.640 \\
\hline Median follow up duration, days (IQR) & 77 & $27-160$ & 123 & $32-262$ & 0.105 \\
\hline \multicolumn{6}{|l|}{ Primary disease } \\
\hline Pancreatic cancer & 15 & $(40.5 \%)$ & 13 & $(35.1 \%)$ & 0.811 \\
\hline Biliary/gallbladder cancer & 5 & $(13.5 \%)$ & 2 & $(5.4 \%)$ & 0.430 \\
\hline Gastric cancer & 5 & $(13.5 \%)$ & 5 & $(13.5 \%)$ & 1.000 \\
\hline Duodenal cancer & 6 & $(16.2 \%)$ & 8 & $(21.6 \%)$ & 0.768 \\
\hline Benign disease & 2 & $(5.4 \%)$ & 2 & $(5.4 \%)$ & 1.000 \\
\hline Breast cancer & 1 & $(2.7 \%)$ & 1 & $(2.7 \%)$ & 1.000 \\
\hline Colorectal cancer & 2 & $(5.4 \%)$ & 0 & $(0.0 \%)$ & 0.493 \\
\hline NET & 1 & $(2.7 \%)$ & 0 & $(0.0 \%)$ & 1.000 \\
\hline Ampullary cancer & 0 & $(0.0 \%)$ & 1 & $(2.7 \%)$ & 1.000 \\
\hline NSCLC & 1 & $(2.7 \%)$ & 1 & $(2.7 \%)$ & 1.000 \\
\hline \multicolumn{6}{|l|}{ Disease stage } \\
\hline Local invasion & 15 & $(40.5 \%)$ & 12 & $(32.4 \%)$ & 0.630 \\
\hline Liver metastases & 6 & $(16.2 \%)$ & 8 & $(21.6 \%)$ & 0.768 \\
\hline Peritoneal metastastases & 6 & $(16.2 \%)$ & 7 & $(18.9 \%)$ & 1.000 \\
\hline Diffuse metastatic & 8 & $(21.6 \%)$ & 6 & $(16.2 \%)$ & 0.768 \\
\hline \multicolumn{6}{|l|}{ Disease manifestations } \\
\hline Ascites & 3 & (8.1\%) & 2 & $(5.4 \%)$ & 1.000 \\
\hline Peritoneal carcinomatosis & 10 & $(27.0 \%)$ & 13 & $(35.1 \%)$ & 0.616 \\
\hline
\end{tabular}

1

2

3 
Table 4. Matched cohort: outcome comparisons.

1

2

\section{EUS-GE \\ $(n=37)$ \\ L-GE \\ $(n=37)$}

\section{Efficacy}

Technical success, n (\%)

Clinical success, $n(\%)$

\author{
35
}

34

(91.9\%)

33

$(100 \%$

(89.2\%)

Per protocol clinical success, $n(\%)$

$34 / 35$

(97.1\%)

(89.2\%)

Median time to oral intake, days (IQR)

Full diet at discharge, $n(\%)$

(0.3-1.0)

(1.0-5.0)

$(56.8 \%)$

(37.8\%)

Median time to full diet, days (IQR)

Gastroenterostomy dysfunction, $\mathrm{n}(\%)$

Distal obstruction, $\mathrm{n}(\%)$

\section{Safety}

Overall adverse events, $\mathrm{n}(\%)$

Mild, n(\%)

Moderate, $\mathrm{n}(\%)$

Severe, $\mathrm{n}(\%)$

Endoscopic reintervention

(1.0-3.8)

(4.0-23)

$(0.0 \%)$

$(0.0 \%)$

$(5.4 \%)$

(2.7\%)
(94.6\%) - 37

\section{OR (95\% Cl), $P$ value}

0.19 (0.01-4.08), 0.493

1.37 (0.29-6.62), 1.000

$4.12(0.44-38.83), 0.358$

$<0.001$

$2.16(0.85-5.46), 0.162$

$<0.001$

1.000

$2.06(0.18-23.72), 1.000$

0.07 (0.01-0.62), 0.007 1.000

$0.13(0.01-2.64), 0.240$

$0.19(0.01-4.08), 0.493$

$0.07(0.00-1.19), 0.025$

$0.13(0.01-2.64), 0.240$

$0.10(0.01-1.91), 0.115$

$\begin{array}{lllll}\text { Anastomotic leak } & 0 & (0.0 \%) & 4 & (10.8 \%)\end{array}$

(5.4\%)

(0.0\%)
$0.19(0.01-4.08), 0.493$

1.000

(0.0\%) $\quad 0$

Fatal, $\mathrm{n}(\%)$

(37.5-80) $\quad 85$

(73.0-110)

$<0.001$ Median hospital stay, days (IQR) (2.0-8.0) $\quad 8$

$<0.001$ Median 2-months' weight change, kg (IQR) $-0,3$ $(-2.4-1.1) \quad 0$

0.159

Median post-procedural survival, days (IQR) 96

(41.5-248) $\quad 152$

(43.5-282)

0.317 


\section{Supplementary tables}

Supplementary table 1 . Sub-analysis comparing $15 \mathrm{~mm}$ and $20 \mathrm{~mm}$ LAMS efficacy and outcomes with or without balloon dilation.

\begin{tabular}{|c|c|c|c|c|c|}
\hline Variable: & $\begin{array}{c}15 \mathrm{~mm} \text { LAMS } \\
(\mathrm{n}=11)\end{array}$ & & $\begin{array}{c}20 \mathrm{~mm} \text { LAMS } \\
(\mathrm{n}=64)\end{array}$ & & $P$-value \\
\hline Technical success, n (\%) & $11 / 11$ & $100 \%$ & $62 / 64$ & $96.9 \%$ & 1.000 \\
\hline Clinical success*, n (\%) & $9 / 11$ & $81.8 \%$ & $62 / 62$ & $100 \%$ & 0.021 \\
\hline Median time to oral intake, days (IQR) & 1 & $(0-1)$ & 1 & $(0-1)$ & 0.826 \\
\hline Full diet tolerability, $\mathrm{n}(\%)$ & 6 & $54.5 \%$ & 45 & $70.3 \%$ & 0.314 \\
\hline Median time to full diet, days (IQR) & 2 & $(1-3)$ & 2 & $(1-4)$ & 0.099 \\
\hline Overall adverse events, n (\%) & 1 & $9.1 \%$ & 4 & $6.5 \%$ & 0.558 \\
\hline Gastroenterostomy dysfunction, n (\%) & 0 & $0.0 \%$ & 1 & $1.6 \%$ & 1.000 \\
\hline Median time to dysfunction, days (IQR) & N/A & N/A & 243 & N/A & N/A \\
\hline Median hospital stay, days (IQR) & 5 & $(2.8-11)$ & 4 & $(2.0-11)$ & 0.054 \\
\hline Median weight change after 2 months, kg (IQR) & $-1,4$ & $(-5.2-0.6)$ & -1 & $(-4.0-1.1)$ & 0.430 \\
\hline Variable: & $\begin{array}{l}\text { With LAMS } \\
\text { dilation }(n=20)\end{array}$ & & $\begin{array}{l}\text { Without LAMS } \\
\text { dilation }(n=55)\end{array}$ & & $P$-value \\
\hline Technical success, n (\%) & $20 / 20$ & $100 \%$ & $53 / 55$ & $96.4 \%$ & 1.000 \\
\hline Clinical success*, n (\%) & $20 / 20$ & $100 \%$ & $51 / 53$ & $96.2 \%$ & 1.000 \\
\hline Median time to oral intake, days (IQR) & 1 & $(0-1)$ & 1 & $(0-1)$ & 0.153 \\
\hline Full diet tolerability, $\mathrm{n}(\%)$ & 10 & $50.0 \%$ & 39 & $70.9 \%$ & 0.107 \\
\hline Median time to full diet, days (IQR) & 2.5 & $(1-4)$ & 2 & $(2-8)$ & 0.254 \\
\hline Overall adverse events, n (\%) & 1 & $5.0 \%$ & 4 & $7.3 \%$ & 0.579 \\
\hline Gastroenterostomy dysfunction, n (\%) & 1 & $5.0 \%$ & 0 & $0.0 \%$ & 1.000 \\
\hline Median time to dysfunction, days (IQR) & 243 & N/A & $\mathrm{N} / \mathrm{A}$ & $\mathrm{N} / \mathrm{A}$ & N/A \\
\hline Median hospital stay, days (IQR) & 5 & $(2.0-11)$ & 4 & $(2.0-11)$ & 0.022 \\
\hline Median weight change after 2 months, kg (IQR) & -1.4 & $(-5.1-0.7)$ & -1 & $(-4.0-1.1)$ & 0.849 \\
\hline
\end{tabular}

Abbreviations: IQR: interquartile range, LAMS: lumen-apposing metal stent, kg: kilogram, N/A: not applicable.

*: per-protocol analysis. 
Supplementary table 2. Multivariate analysis of variables in EUS-GE clinical failure.

1

2

\begin{tabular}{lccc}
\hline Variable & OR & $95 \% \mathrm{Cl}$ & $P$-value \\
\hline Age & 0.98 & $0.90-1.03$ & 0.532 \\
Gender, female & 2.95 & $0.46-11.18$ & 0.132 \\
Pancreatic cancer & 3.14 & $0.69-20.22$ & 0.115 \\
Ascites & 8.39 & $1.29-375.4$ & 0.104 \\
Peritoneal carcinomatosis & 2.65 & $0.44-28.94$ & 0.305 \\
15mm LAMS & 2.45 & $0.14-14.09$ & 0.332 \\
Balloon dilation & 0.11 & $0.01-0.72$ & 0.059 \\
Learning curve, first 50\% & 1.30 & $0.37-15.49$ & 0.726 \\
\hline
\end{tabular}

Supplementary table 3. Learning curve assessment: comparison between first and second half of the EUS-GE cohorts.

\begin{tabular}{|c|c|c|c|c|c|}
\hline Propensity score-matched EUS-GE cohort: & $\begin{array}{l}\text { First } 50 \% \\
\quad(n=18)\end{array}$ & & $\begin{array}{l}\text { Second } 50 \% \\
\quad(n=19)\end{array}$ & & $P$-value \\
\hline Technical success, n (\%) & $17 / 18$ & $94.4 \%$ & $18 / 19$ & $94.7 \%$ & 1.000 \\
\hline Clinical success, n (\%) & $17 / 18$ & $94.4 \%$ & $17 / 19$ & $89.5 \%$ & 1.000 \\
\hline Per protocol clinical success, $\mathrm{n}(\%)$ & $17 / 17$ & $100 \%$ & $17 / 18$ & $94.4 \%$ & 1.000 \\
\hline Overall adverse events, $\mathrm{n}(\%)$ & 1 & $5.6 \%$ & 1 & $5.3 \%$ & 1.000 \\
\hline Median procedure duration, $\min (I Q R)$ & 51 & $(34-84)$ & 46 & $(40-83)$ & 0.719 \\
\hline Overall EUS-GE cohort: & $\begin{array}{c}\text { First } 50 \% \\
(n=38)\end{array}$ & & $\begin{array}{l}\text { Second } 50 \% \\
\quad(n=39)\end{array}$ & & $P$-value \\
\hline Technical success, $\mathrm{n}(\%)$ & $37 / 38$ & $97.4 \%$ & $36 / 39$ & $92.3 \%$ & 0.615 \\
\hline Clinical success, $n(\%)$ & $37 / 38$ & $97.4 \%$ & $34 / 39$ & $87.2 \%$ & 0.200 \\
\hline Per protocol clinical success, n (\%) & $37 / 37$ & $100 \%$ & $34 / 36$ & $94.4 \%$ & 0.240 \\
\hline Overall adverse events, n (\%) & 3 & $7.9 \%$ & 3 & $7.7 \%$ & 1.000 \\
\hline Median procedure duration, $\min (\mathrm{IQR})$ & 49 & $(36-78)$ & 52 & $(33-83)$ & 0.719 \\
\hline
\end{tabular}

Abbreviations: EUS-GE: Endoscopic ultrasound-guided gastroenterostomy, IQR: interquartile range. 

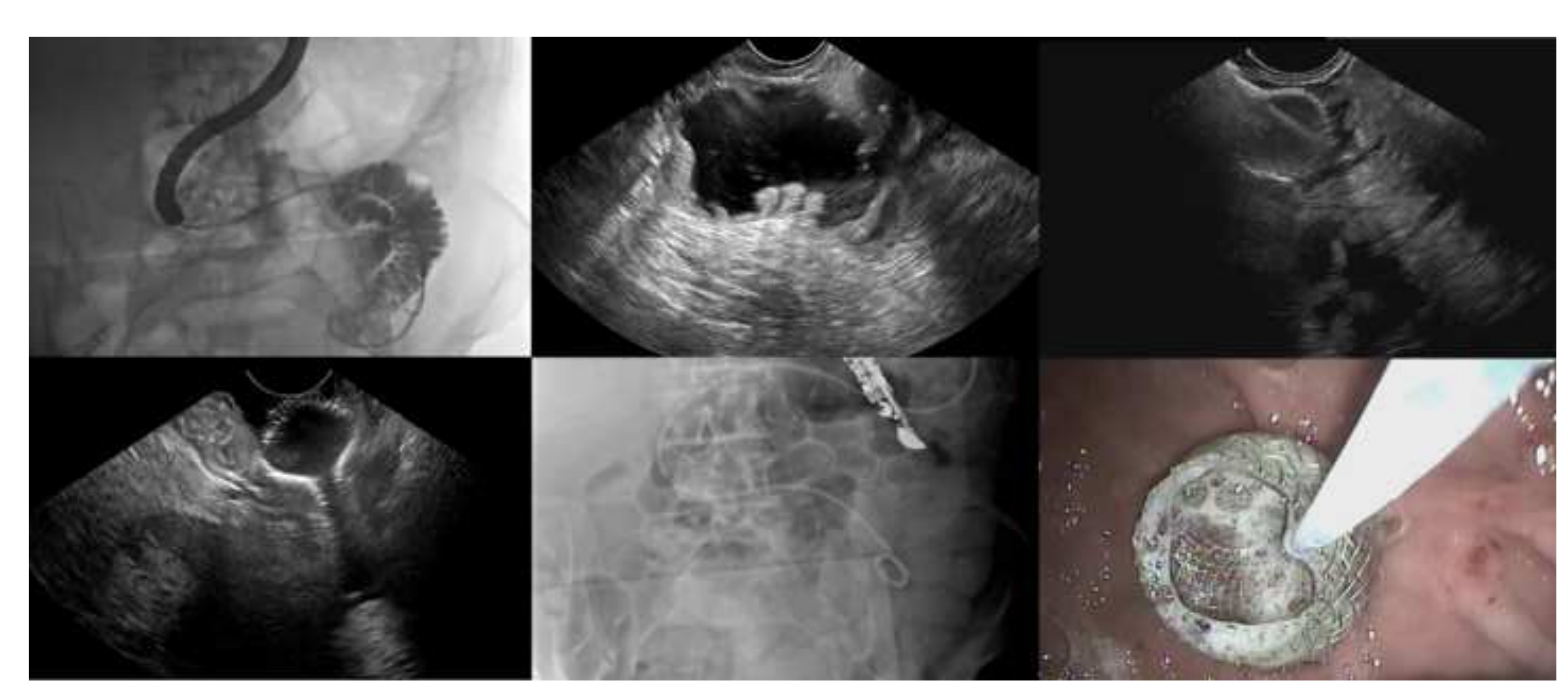


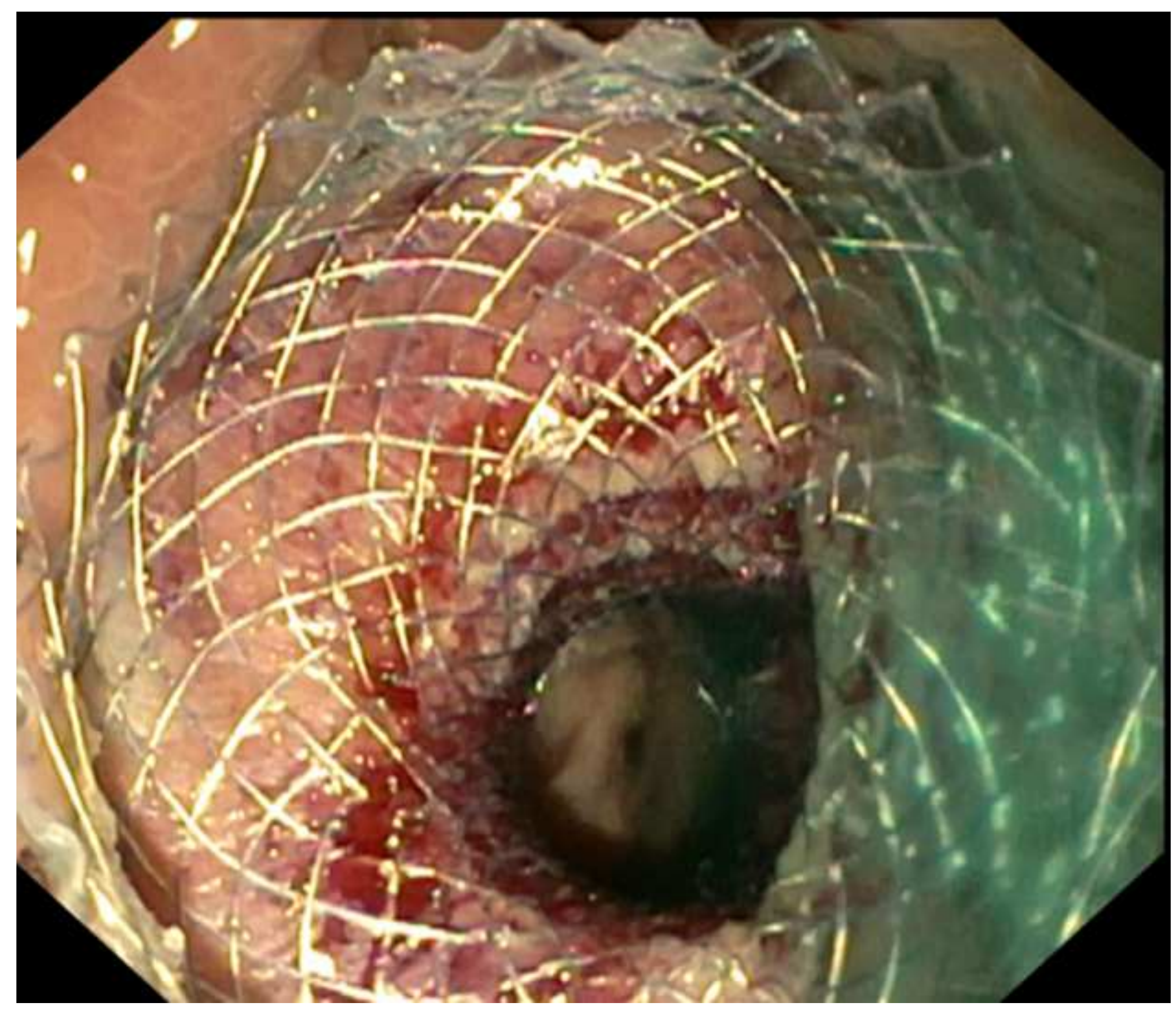



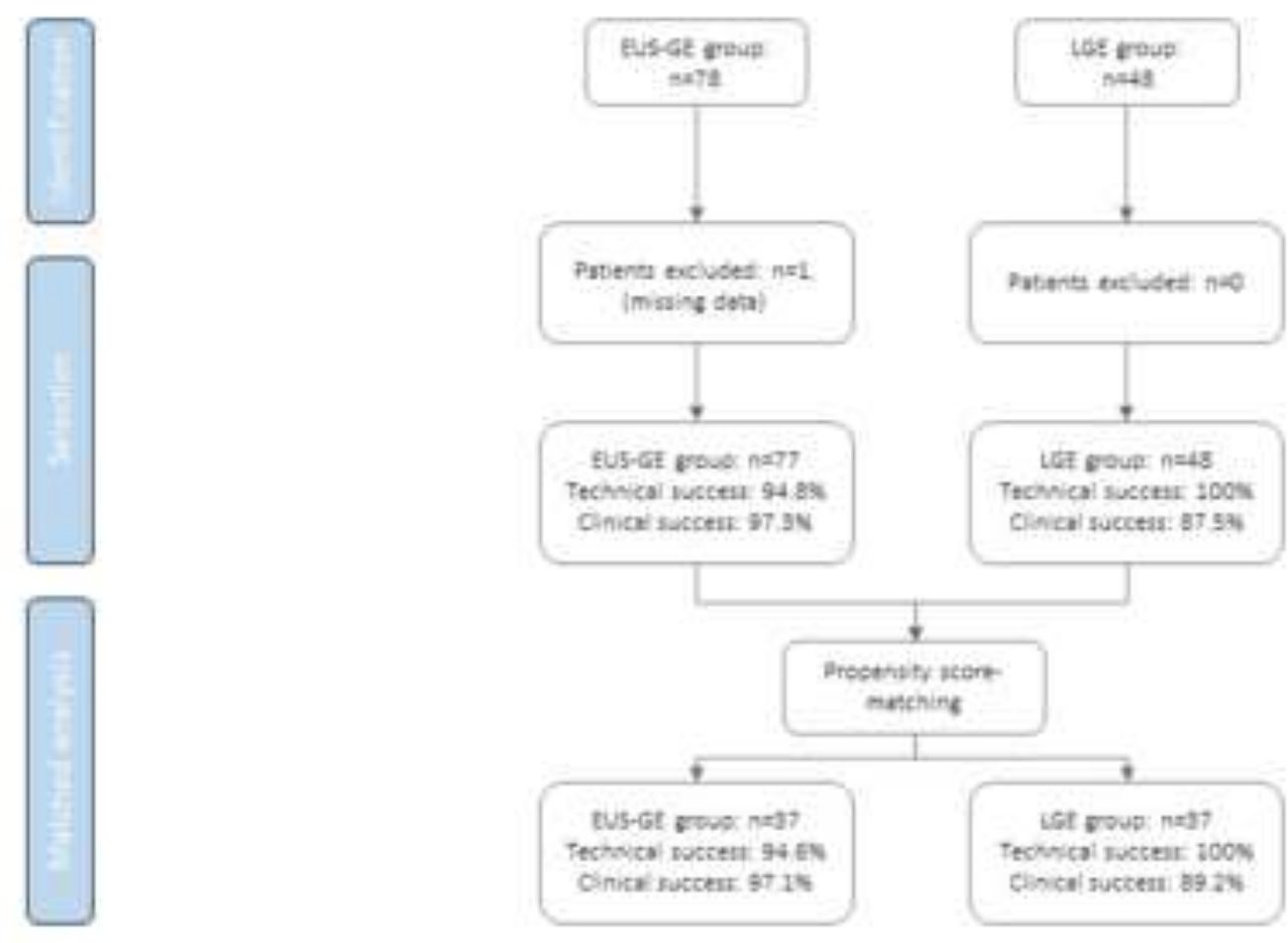


\section{Matched Cohort}

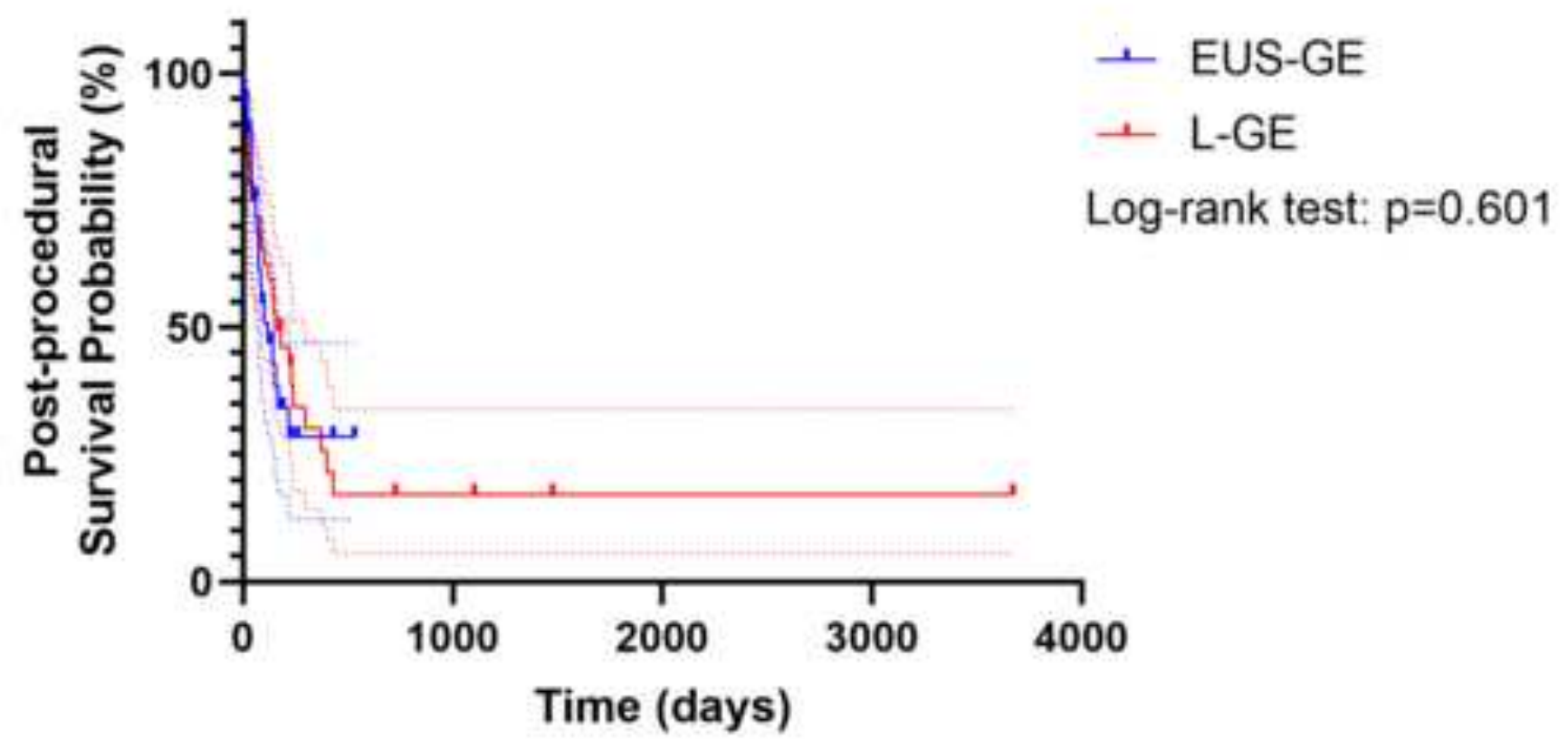

Overall Cohort

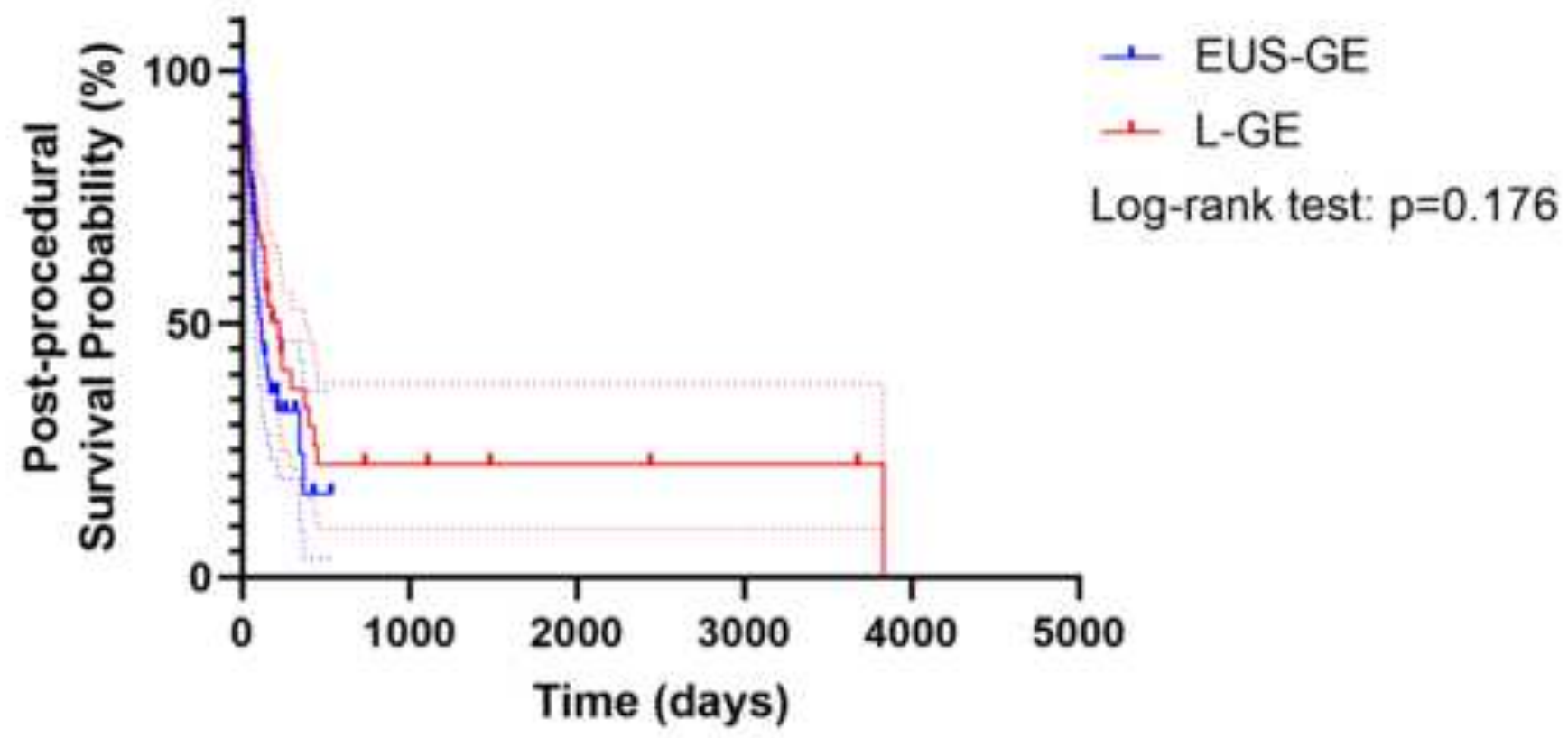


Click here to access/download Video Component(s) (each less than 100 MB) WEST_Video.wmv 
Acronyms and abbreviations (list all that are used in paper with their spell-outs

Laparoscopic versus EUS-guided Gastroenterostomy for Gastric Outlet Obstruction: An International Multicentre Propensity Score-Matched Comparison.

Abbreviations:

AE: adverse event

ASGE: American society gastrointestinal endoscopy

$\mathrm{Cl}$ : confidence interval

EUS: endoscopic ultrasound

EUS-GE: EUS-guided gastroenterostomy

GOO: gastric outlet obstruction

IQR: interquartile range

LAMS: lumen-apposing metal stent

L-GE: laparoscopic gastroenterostomy

OR: odds ratio

SD: standard deviation

\author{
SEMS: self-expandable metal stents
}




\section{GIE IRB Checklist}

Note that GIE follows the International Committee of Medical Journal Editors (ICMJE)'s Uniform Requirement for Manuscripts Submitted to Biomedical Journals. All clinical trials submitted to GIE should have been registered BEFORE the trial begins through one of the registries approved by the ICMJE, and proof of that registration, including the date registered and the registration number, must be submitted to GIE along with the article. IRBH approval information must be included in the manuscript text, including the date of registration. All clinical trials as defined by the ICMJE must also have been registered before the trial began (not just randomized clinical trials).

\begin{tabular}{|l|l|}
\hline & $\begin{array}{l}\text { Have you included IRB information in your article? If not, please explain why. Do } \\
\text { not merely put NA or your article will be returned for a fuller explanation. } \\
\text { Comments: Yes, we've included this in the Methods-section. }\end{array}$ \\
\hline Please list here the DATE of IRB approval for your study. \\
$\begin{array}{l}\text { Date of approval: August } 6^{\text {th }} \text { 2020. Central IRB: University Hospitals Leuven. } \\
\text { Proof of registration for randomized clinical trials, including registration number } \\
\text { the trial was started, is required before subject enrollment; have you included } \\
\text { this information? } \\
\text { No, this was a retrospective study. No trial registration was therefore required. }\end{array}$ \\
\hline
\end{tabular}




\section{Journal CME Conflict of Interest: Disclosure and Attestation}

\section{Lead Author: Michiel Bronswijk, MD \\ Laparoscopic versus EUS-guided Gastroenterostomy for Gastric Outlet Article: Obstruction: An International Multicentre Propensity Score-Matched Comparison.}

\section{Date: November $19^{\text {th }} 2020$}

The purpose of this form is to identify all potential conflicts of interests that arise from financial relationships between any author for this article and any commercial or proprietary entity that produces healthcare-related products and/or services relevant to the content of the article. This includes any financial relationship within the last twelve months, as well as known financial relationships of authors' spouse or partner. The lead author is responsible for submitting the disclosures of all listed authors, and must sign this form at the bottom. Additional forms may be submitted if the number of authors exceeds the space provided.

\section{Lead Author: Michiel Bronswijk}

Email Address*: mjh.bronswijk@gmail.com

No financial relationships with a commercial entity producing health-care related products and/or services relevant to this article.

\begin{tabular}{|l|l|c|}
\hline \multicolumn{1}{|c|}{ Company } & \multicolumn{1}{|c|}{ Type of Relationship** } & Content Area (if applicable) \\
\hline Taewoong & Travel grants & \\
\hline Prion Medical & Travel grants & \\
\hline Takeda & Travel grants & \\
\hline
\end{tabular}

\section{Author: Giuseppe Vanella}

Email Address*: g.e.vanella@gmail.com

No financial relationships with a commercial entity producing health-care related products and/or services relevant to this article.

\section{Author: Hannah van Malenstein}

Email Address*: hannah.vanmalenstein@uzleuven.be

No financial relationships with a commercial entity producing health-care related products and/or services relevant to this article.

\begin{tabular}{|l|l|c|}
\hline \multicolumn{1}{|c|}{ Company } & Type of Relationship** & Content Area (if applicable) \\
\hline Boston scientific & Consultancy agreement & \\
\hline
\end{tabular}

\section{Author: Wim Laleman}

Email Address*: wim.laleman@uzleuven.be

No financial relationships with a commercial entity producing health-care related products and/or services relevant to this article.

\begin{tabular}{|l|l|l|}
\hline \multicolumn{1}{|c|}{ Company } & \multicolumn{1}{c|}{ Type of Relationship** } & \multicolumn{1}{c|}{ Content Area (if applicable) } \\
\hline Boston scientific & Consultancy agreement & \\
\hline Cook & Consultancy agreement & $\begin{array}{l}\text { Co-chair in Therapeutic HPB } \\
\text { endoscopy }\end{array}$ \\
\hline Boston scientific & & \\
\hline
\end{tabular}

\footnotetext{
* We will use email addresses only for questions related to this article 1

** Type of relationship may include: full-time or part-time employee, independent contractor, consultant, research or other grant recipient, paid speaker or teacher, membership on advisory committee or review panels, ownership interest (product royalty/licensing fees, owning stocks, shares, etc.), relationship of a spouse or partner, or any other financial relationship.
} 
Author: Joris Jaekers

$\bigotimes$

\section{Author: Baki Topal}

$\bigotimes$

\section{Author: Freek Daams}

\section{Email Address*: joris.jaekers@uzleuven.be}

No financial relationships with a commercial entity producing healthcare related products and/or services relevant to this article.

\section{Email Address*: baki.topal@uzleuven.be}

No financial relationships with a commercial entity producing healthcare related products and/or services relevant to this article.

Email Address*: f.daams@amsterdamumc.nl

No financial relationships with a commercial entity producing health-care related products and/or services relevant to this article.

Author: Mark Besselink

\section{Email Address*: m.besselink@amsterdamumc.nl}

No financial relationships with a commercial entity producing health-care related products and/or services relevant to this article.

\begin{tabular}{|l|l|l|}
\hline \multicolumn{1}{|c|}{ Company } & \multicolumn{1}{|c|}{ Type of Relationship** } & Content Area (if applicable) \\
\hline Ethicon & Research grants & \\
\hline Medtronic & Research grants & \\
\hline Intuitive & Research grants & \\
\hline
\end{tabular}

Author: Paolo Giorgio Arcidiacono

Email Address*: arcidiacono.paologiorgio@hsr.it

No financial relationships with a commercial entity producing health-care related products and/or services relevant to this article.

\section{Author: Rogier Voermans}

Email Address*: r.voermans@amsterdamumc.nl

No financial relationships with a commercial entity producing health-care related products and/or services relevant to this article.

\begin{tabular}{|l|l|l|}
\hline \multicolumn{1}{|c|}{ Company } & Type of Relationship** & Content Area (if applicable) \\
\hline Boston scientific & Consultancy fees and research grants & \\
\hline & & \\
\hline
\end{tabular}

\section{Author: Paul Fockens}

$\bigotimes$

\section{Email Address*: p.fockens@amsterdamumc.nl}

No financial relationships with a commercial entity producing health-care related products and/or services relevant to this article.

\section{Author: Alberto Larghi}

Email Address*: alberto.larghi@yahoo.it

No financial relationships with a commercial entity producing health-care related products and/or services relevant to this article.

\section{Author: Roy van Wanrooij}

\section{Email Address*: rl.vanwanrooij@amsterdamumc.nl} No financial relationships with a commercial entity producing health-care related products and/or services relevant to this article.

\footnotetext{
* We will use email addresses only for questions related to this article 2

${ }^{* *}$ Type of relationship may include: full-time or part-time employee, independent contractor, consultant, research or other grant recipient, paid speaker or teacher, membership on advisory committee or review panels, ownership interest (product royalty/licensing fees, owning stocks, shares, etc.), relationship of a spouse or partner, or any other financial relationship.
} 
No financial relationships with a commercial entity producing health-care related products and/or services relevant to this article.

\begin{tabular}{|l|l|l|}
\hline \multicolumn{1}{|c|}{ Company } & \multicolumn{1}{|c|}{ Type of Relationship** } & Content Area (if applicable) \\
\hline Cook & Consultancy agreement & \\
\hline Pentax & Consultancy agreement & \\
\hline Olympus & Consultancy agreement & Co-chair HPB endoscopy \\
\hline Boston scientific & & Conter \\
\hline
\end{tabular}

As corresponding author of this article, I attest that I have received disclosure information from all participating authors as listed above and acknowledge that I am responsible for verifying the accuracy of and reporting completely the information provided to me. Financial relationships relevant to this article can be researched at https://www.cms.gov/openpayments/. I understand that typing my name below serves as an electronic signature for the purposes of this form.

\section{Schalk van der Merwe, MD MSc PhD}

Type Name (Electronic Signature) 